\title{
Maximizing bandgap width and in-plane stiffness of porous phononic plates for tailoring flexural guided waves: topology optimization and experimental validation
}

\author{
Saeid Hedayatrasa ${ }^{1, *}$, Mathias Kersemans ${ }^{2}$, Kazem Abhary ${ }^{1}$, Mohammad Uddin ${ }^{1}$, James K. \\ Guest $^{3}$ and Wim Van Paepegem ${ }^{2}$ \\ ${ }^{1}$ School of Engineering, University of South Australia, Mawson Lakes, SA 5095, Australia \\ ${ }^{2}$ Department of Materials Science and Engineering, Ghent University, Technologiepark- \\ Zwijnaarde 903, 9052 Zwijnaarde, Belgium \\ ${ }^{3}$ Department of Civil Engineering, Department of Materials Science and Engineering, Johns \\ Hopkins University, Baltimore, MD 21218-2686, USA
}

\begin{abstract}
Phononic crystal plates (PhPs) with porous heterogeneities manufactured through perforation of a uniform plate comprise effective wave reflecting interfaces and are free from complexities and interfacial imperfections associated with fabricating bi-material designs. However, numerical optimization of such porous $\mathrm{PhPs}$ for maximized bandgap efficiency naturally leads to topologies with isolated solid domains, or disconnected island-like features. Requiring PhPs to exhibit an adequate stiffness is, therefore, an important design consideration. This paper presents a multi-objective topology optimization study with experimental validation to explore the relationship between bandgap efficiency and effective in-plane stiffness, introducing topologies exhibiting superior properties compared to those reported in earlier works. Topology optimization is performed in two stages: first, at a relatively coarse resolution followed by a topology optimization on a refined mesh. The minimum allowable length scale of features is maintained across the mesh refinement, and thus the refined stage primarily leads to optimization of the feature boundaries, which is shown to significantly enhance response. Bandgap of fundamental flexural guided wave modes is studied herein, and it is shown how bandgap efficiency is degraded by increasing the in-plane stiffness. Distinct stiff and compliant topology modes are realized in the intermediate section of the optimized Pareto fronts which offer variation of structural stiffness while having almost the same bandgap efficiency, with potential application as base cells in design of gradient phononic lattices. A subset of promising stiff and compliant optimized topologies are manufactured by water-jetting of an aluminum plate and experimentally tested to validate the calculated bandgap efficiencies and effective elastic properties.
\end{abstract}

\footnotetext{
* Corresponding author. Tel. +61883026954

Email address: saeid.hedayatrasa@mymail.unisa.edu.au (S. Hedayatrasa)
} 
Keywords: Topology Optimization; Experimental validation; Phononic Crystal; Plate; Stiffness; Water-jet

\section{Introduction}

Extraordinary features of heterogeneous lattice structures in controlling acoustic and elastodynamic waves have attracted a great deal of research and motivated development of design optimization methods. The destructive interaction of waves within these structures when the wavelength is comparable to the lattice periodicity, enables self-collimation, negative refraction and even total reflection of particular frequencies (Deymier 2011; Lin 2012; NematNasser 2015a; Park, Ma \& Kim 2015). Frequency ranges over which successive in-phase (Bragg) reflection of waves at the interface of periodic heterogeneities causes exponential decay of the wave amplitude are called phononic bandgaps. The width of bandgap and its frequency range depend on the acoustic mismatch of constitutive materials, lattice shape and periodicity and essentially topology of its irreducible periodic feature (unit-cell)(Sigmund \& Jensen 2003). Some features may behave as an internal oscillator and open (or widen) bandgaps at their local resonance frequency which may manipulate wavelengths considerably larger than the lattice periodicity (Liu et al. 2000; Krushynska, Kouznetsova \& Geers 2014; Hedayatrasa, Abhary \& Uddin 2016). For specified unit-cell size, the widest bandgap at lowest frequency range is generally desired. In this way the tiniest feature for manipulating a particular wavelength is achieved. Hence the relative bandgap width (RBW), defined as the ratio of bandgap width over mid-gap frequency, should be maximized during topology optimization.

Phononic crystal plates (PhPs) have promising application in manipulation of guided waves in plate structures and are applicable for design of low-loss acoustic devices and built-in acoustic metamaterial lenses (Olsson Iii et al. 2008; Zhu \& Semperlotti 2014). The resultant waves guided through plate structures have symmetric and asymmetric polarizations with respect to the mid-plane, known as: symmetric Lamb (S) and shear horizontal (SHS) wave modes, and asymmetric Lamb (A) and shear horizontal (SHA) wave modes. The asymmetric modes A and SHA are associated with flexural deformation of the plate, so-called flexural (or bending) waves. Flexural modes are predominantly excited through an arbitrary lateral disturbance, carry a large portion of wave energy and are efficiently coupled with acoustic waves. An exclusive bandgap of flexural waves can be opened between first two asymmetric modal branches at a frequency range considerably lower than the complete bandgap of mixed wave modes. This means larger incident wavelengths (i.e. lower frequencies) can be controlled by smaller feature sizes.

Phononic lattices can be fabricated by integrating two or more materials (Hussein et al. 2007; Hedayatrasa, Abhary \& Uddin 2015; Nemat-Nasser 2015b; Guo, Wei \& Li 2016), or 
through a single material porous design (Bilal \& Hussein 2011; Dong, Su \& Wang 2014; Delpero et al. 2016; Hedayatrasa et al. 2016a; Hedayatrasa et al. 2016b). Single material porous designs are relatively easy to produce (compared to multiple-materials) and are free from complexities and interfacial imperfections associated with fabricating bi-material designs. Moreover, the porosities provide a perfect acoustic mismatch with solid matrix and are effective in reflecting the wave energy. Porous 2D PhP can be produced by periodic perforation of a uniform base plate, with no through thickness gradient e.g. (Wang \& Wang 2013; Celli \& Gonella 2014). In this case, the $2 \mathrm{D}$ topology optimization may be used to search for the best perforation profile of the $\mathrm{PhP}$ unit-cell.

Gradient-based optimization is known to be an effective approach to solving high-resolution topology optimization problems. In contrast, stochastic optimization algorithms like genetic algorithms (GA) are very sensitive to the number of design variables. The population size of GA should be proportionally increased with respect to the number of design variables to get reliable optimized results, leading to an extremely increased computational cost for high resolutions. However, topology optimization of porous phononic bandgaps has particular challenges:

- When maximizing RBW, the optimization algorithm tends to maximize the interfacial reflections and naturally a compliant topology with extraordinary small features is obtained. This can be circumvented by imposing a minimum feature size and/or incorporating effective stiffness constraints or objectives in optimization algorithm.

- In gradient-based optimization of porous structures, it is typical to assign a relatively compliant material to the void regions to maintain the continuity of the design domain and avoid singularities. This assumption, when maximizing the RBW, leads to appearance of infeasible isolated solid features, or islands, even if the stiffness and solid feature sizes are controlled. In fact, any isolated segment is coupled with the solid matrix through the compliant material assigned to voids and therefore artificially contributes to widening bandgap through Bragg scattering or local resonance. This misleads the sensitivity analysis and compromises the optimality of any output topologies, which would be reduced to connected features (if any).

Halkjær, Sigmund and Jensen (2006) performed gradient based topology optimization of porous 2D PhPs with rhombic unit-cell and width to thickness (aspect ratio) of 11 for maximized RBW of the first couple of asymmetric modal branches. The optimization was not successful in terms of finding a feasible topology without discontinuities and an alternative feasible topology was introduced after post-processing. 
In a further work by Bilal and Hussein (2012) GA was employed for optimization of 2D PhP but with square symmetry, and a feasible topology was obtained with higher RBW as compared with that of Halkjær, Sigmund and Jensen (2006). The domain was discretized and optimized at a resolution of $32 \times 32$ pixels and finally refined to $64 \times 64$. The discreteness of GA provides the capability to check the feasibility of topology and reject or penalize discontinuous topologies. Thus the topology achieved by this later work did not require any post-processing for feasibility.

In a recent work by the authors (Hedayatrasa et al. 2016b) multiobjective GA was employed for optimization of thin and thick porous 2D PhPs with respect to homogenized in-plane stiffness, in order to take into account the stiffness of porous topologies. Specific operations were implemented to treat the discontinuities of randomly generated candidate topologies in a random progressive manner. Complete bandgaps of mixed wave modes and exclusive bandgaps of symmetric and flexural modes were studied separately. A square symmetric unit-cell with resolution of $32 \times 32$ was assumed and improved bandgaps of flexural waves were obtained compared with earlier works (Halkjær, Sigmund \& Jensen 2006; Bilal \& Hussein 2012). A compliant material was used as the void phase for simplicity in evaluating randomly generated topologies.

Although many works have been dedicated to the topology optimization of phononic bandgaps, their experimental study has been very limited (Halkjær, Sigmund \& Jensen 2006). This paper is an extension of preceding work by the authors (Hedayatrasa et al. 2016b) with a focus on optimization and new experimental validation of exclusive bandgaps of flexural guided waves in thin PhPs, including the following contributions:

- An improved and more efficient topology optimization algorithm is implemented, in which relatively coarse and fine topology resolutions are optimized sequentially. Relevant Pareto fronts are detailed and compared to demonstrate their relative efficiency. Distinct stiff and compliant topology modes are realized which offer significant increase in the stiffness with almost the same RBW.

- Refined topologies are achieved with considerably broader bandgap-stiffness efficiency combinations than reported in previous works (Halkjær, Sigmund \& Jensen 2006; Bilal \& Hussein 2012; Hedayatrasa et al. 2016b).

- A subset of promising compliant and stiff topologies are manufactured and their bandgapstiffness efficiencies are validated experimentally.

Square symmetric 2D PhPs of flexural waves with aspect ratio of 10 are optimized with relatively coarse and fine topology resolutions of $32 \times 32$ and $64 \times 64$, respectively. Elements 
associated with void regions of the topology are removed from the model before analysis for faster and more accurate fitness evaluation, meaning that no compliant filling material is needed. The topology is first optimized using the coarse resolution with the optimized solution then used to seed the more refined topology optimization performed at a resolution of $64 \times 64$ for an additional number of generations.

Aluminum PhPs of selected optimized topologies (of both resolutions) are water-jetted and experimentally validated. The transmission of excited signals at the uniform boundary to specific points of PhPs are measured by both piezoelectric transducers (PZTs) and laser Doppler vibrometer (LDV), and relevant spectra are verified with calculated modal band structures. Furthermore, the effective stiffness of produced $\mathrm{PhP}$ specimens of refined topologies is experimentally investigated through tensile tests with deformation measured by (i) mechanical extensometers and (ii) stereovision digital image correlation (3D-DIC).

The layout of the paper is as follows. The optimization objectives, constitutive equations, and implemented multiobjective optimization algorithm are first discussed. Then the obtained Pareto front and selected optimized PhP topologies are presented and explained. Finally, the produced $\mathrm{PhP}$ designs and experimental evaluation of their bandgap efficiency and effective stiffness are presented and discussed.

\section{Topology optimization of $\mathbf{P h P}$}

A relatively thin 2D PhP unit-cell as shown in in Figure 1(a) with aspect ratio (width to thickness) $a / h=10$ is considered to be produced by through thickness perforation of a uniform background plate. Square symmetry is prescribed which leads to an orthotropic phononic lattice plate with in-plane symmetry.

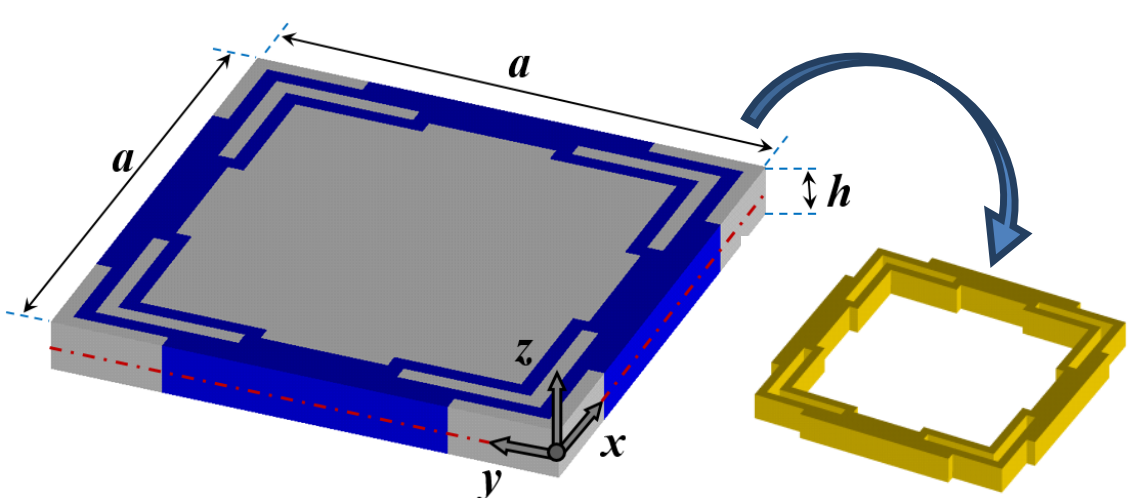

(a)

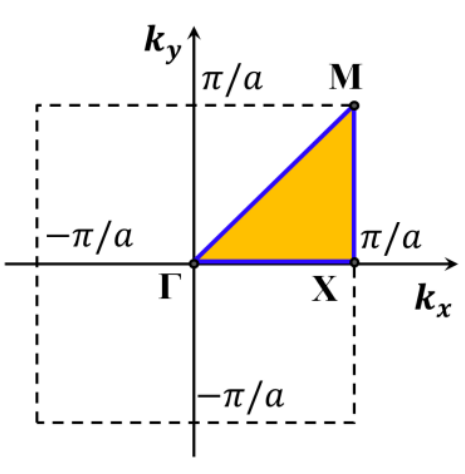

(b)

Figure 1 (a) Schematic of assumed square symmetric thin PhP unit-cell with aspect ratio $a / h=10$ for an arbitrary perforation profile (light area), and (b) relevant irreducible Brillouin zone ГХМ 
Aluminum with elastic modulus $E_{s}=70 \mathrm{GPa}$, Poisson's ratio $v_{s}=0.34$ and density $\rho_{s}=2700 \mathrm{~kg} / \mathrm{m}^{3}$ is considered for the constitutive material of porous $\mathrm{PhP}$ and the unit-cell is explored to achieve the following two objectives:

1. Maximized RBW between the first two asymmetric modal branches to obtain lowest bandgap frequency range, and/or

2. Maximized effective in-plane stiffness.

The finite element method (FEM) is implemented through ANSYS APDL FEM solver (ANSYS® Academic Research, Release 16) for fitness evaluation of candidate topologies for both objectives. 3D solid-shell elements SOLSH190 are used for analysis of relatively thin unitcells of aspect ratio 10, and only the solid domain of topology is modelled and analyzed during optimization, as discussed later in Section 2.2.

\subsection{Objective functions}

The first objective of the optimization is to maximize RBW of the first two asymmetric modal branches. In order to define the modal band structure of the candidate topology, the modal frequencies $\omega$ of the unit-cell are calculated for various in-plane wave vectors $\mathbf{k}=\left[\begin{array}{ll}k_{x} & k_{y}\end{array}\right]$ on the border of irreducible Brillouin zone as shown by triangular area ГХМ in Figure 1(b) for the square symmetric topology. The FEM notation of the equation of motion after substituting the Bloch wave equation (Kittel 1986) is:

$$
\left(-\mathbf{M} \omega^{2}+\mathbf{K}(\mathbf{k})\right) \mathbf{q}=\mathbf{0}
$$

where $\mathbf{M}$ is the mass matrix, $\mathbf{K}$ is the stiffness matrix, $\mathbf{q}$ is the vector of nodal displacements and $\omega=2 \pi f$ is the circular frequency. The wave vector $\mathbf{k}$ is prescribed through applying a phase delay between the oscillations of opposite periodic boundaries of the unit-cell (Aberg \& Gudmundson 1997) as follows:

$$
\mathbf{u}(\mathbf{x}, t)=\mathbf{u}(\mathbf{x}+\mathbf{A}, t) e^{i \mathbf{k} \cdot \mathbf{A}}
$$

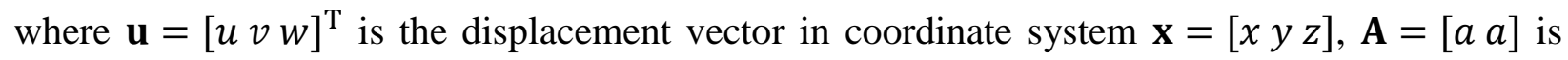
the lattice periodicity vector of the square $2 \mathrm{D} \mathrm{PhP}$ with unit-cell width $a, t$ is time and $i=\sqrt{-1}$. By calculation of the modal band structure over the $n_{k}$ discrete search points $\mathbf{k}_{i}$ on the border of the irreducible Brillouin zone, the first objective function to be maximized can be defined as:

$$
F_{1}=\frac{\min _{i=1}^{n_{k}} \omega_{j+1}^{2}\left(\mathbf{k}_{i}\right)-\max _{i=1}^{n_{k}} \omega_{j}^{2}\left(\mathbf{k}_{i}\right)}{0.5\left(\min _{i=1}^{n_{k}} \omega_{j+1}^{2}\left(\mathbf{k}_{i}\right)+\max _{i=1}^{n_{k}} \omega_{j}^{2}\left(\mathbf{k}_{i}\right)\right)}
$$

which is the bandgap width over mean value of the gap between eigenvalues of first consecutive modal branches $j=1$ and $j+1=2$. In order to decouple the asymmetric modes of the $\mathrm{PhP}$ 
unit-cell, only half of the unit-cell from mid-plane $z=0$ to top plane $z=h / 2$ (Figure 1(a)) is modelled and in-plane displacement of the mid-plane is constrained $\left(\left.\{u, v\}\right|_{z=0}=0\right)$ (Hedayatrasa et al. 2016b).

The effective strain energy of the unit-cell under in-plane loading is defined as the second objective to be minimized, in order to maximize the effective in-plane stiffness of the PhP unitcell. Due to the direct contribution of in-plane stresses to the through-thickness resistive moments of a plate, higher in-plane stiffness leads to higher overall flexural stiffness as well (Timoshenko \& Woinowsky-Krieger 1959). This is particularly valid for the assumed upright perforated unit-cell with no possibility of through thickness topology and material gradient to control flexural properties.

The strain energy density at a point subjected to in-plane stress in $\mathrm{x}-\mathrm{y}$ plane is defined as:

$$
U_{V}=\frac{1}{2}\left(\sigma_{x} \varepsilon_{x}+\sigma_{y} \varepsilon_{y}+\tau_{x y} \gamma_{x y}\right)
$$

where $\left\{\sigma_{x}, \sigma_{y}, \tau_{x y}\right\}$ and $\left\{\varepsilon_{x}, \varepsilon_{y}, \gamma_{x y}\right\}$ are corresponding in-plane stress and strain vectors, respectively. By prescribing the in-plane stress state $\left\{\sigma_{x}, \sigma_{y}, \tau_{x y}\right\}=\{\sigma, \sigma, b \sigma\}$ and with regards to linear elastic stress-strain equations, $U_{V}$ is reformulated as:

$$
U_{V}=\sigma^{2}\left(\frac{1-v_{e}}{E_{e}}+\frac{0.5 \mathrm{~b}^{2}}{G_{e}}\right)
$$

where $E_{e}, G_{e}$ and $v_{e}$ are the effective in-plane orthotropic elastic modulus, shear modulus and Poisson's ratio of the assumed square symmetric $\mathrm{PhP}$ unit-cell, respectively. Therefore the second objective function $F_{2}$ is defined as the relative strain energy compliance of the unit-cell, based on its effective properties, to be minimized:

$$
F_{2}=\frac{1}{\epsilon_{s}}\left(\left(\frac{1-v_{e}}{E_{e}}\right)+\left(\frac{1}{G_{e}}\right)\right)
$$

where constant $\epsilon_{\mathrm{s}}$ is the compliance of pure solid matrix:

$$
\epsilon_{\mathrm{s}}=\left(\frac{1-v_{s}}{E_{s}}\right)+\left(\frac{1}{G_{s}}\right)
$$

The relative magnitude of normal and shear stresses is assumed to be $b=\sqrt{2}$ which introduces a stiffness objective function with equal contribution of normal and shear compliances (the first and the second terms of Eq.6, respectively). In an earlier study by the authors (Hedayatrasa et al. 2016b) it was shown that the relative dominance of shear or normal stiffness can be controlled by the ratio $b$, leading to distinct sets of optimized topologies. Expectedly, a ratio of $b=0$ (i.e. 
$<\sqrt{2}$ ) led to dominant normal stiffness, and a ratio of $b=2$ (i.e. $>\sqrt{2}$ ) led to dominant shear stiffness.

Computational homogenization is performed through FEM to calculate the effective elastic properties of the $\mathrm{PhP}$ unit-cell and periodic boundary conditions are applied to ensure periodicity of the unit-cell (Steven 2006). Pure shear and pure normal test strains are applied individually to a single representative unit-cell and the effective elastic properties are calculated based on volumetric average of the stress field over the entire unit-cell.

\subsection{Multiobjective topology optimization approach}

The original optimization algorithm based on non-dominated sorting genetic algorithm (NSGA-II) (Pratap, Agarwal \& Meyarivan 2002) used in the earlier study (Hedayatrasa et al. 2016b) is employed with few improvements for more efficient fitness evaluation and topology evolution.

A square symmetric topology as shown in Figure 2(a) is assumed in which the highlighted one-eighth section is the independent design domain. Topology optimization is performed in two subsequent stages first with coarse resolution $32 \times 32$ and then refined resolution $64 \times 64$. Initiating the optimization by the much bigger design space of resolution $64 \times 64$ would impose a very high computational cost for a reliable optimization. The independent design variables are assigned to element centroids of the coarse topology (Figure 2(b)) and are then mapped into nodal design variables of refined topology (Figure 2(c)) when moving into the second stage. The refined topology uses a small projection radius of one element size during optimization to maintain a consistent minimum length scale with the coarse stage design while still allowing small shifts in topology (Guest \& Smith Genut 2010).

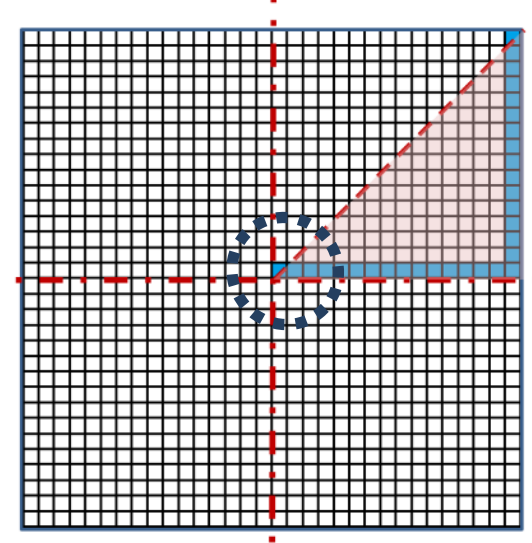

(a)

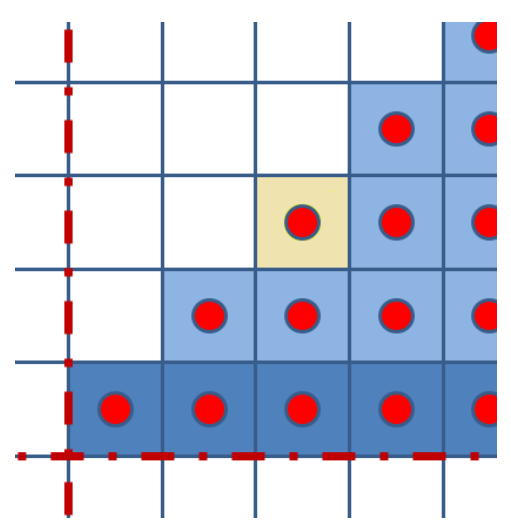

(b)

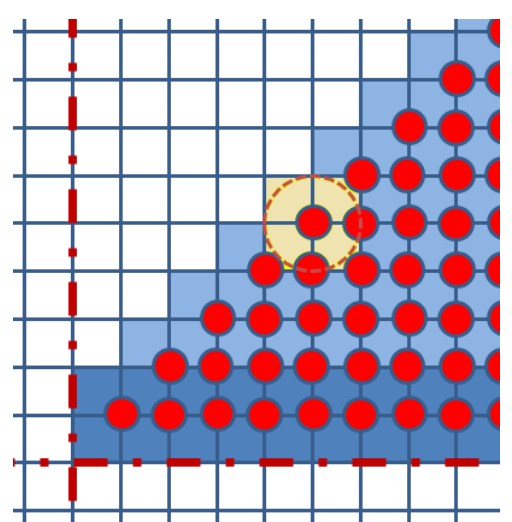

(c)

Figure 2 (a) Square symmetric topology with coarse resolution of $32 \times 32$, (b) close view of relevant design variables on element centroids, and (c) nodal design variables assigned to the refined topology resolution of $64 \times 64$ to be projected into 4 adjacent elements 
The boundary nodes are preferably excluded from the design variable field in order to limit the radius of orthogonal interconnecting elements of topology (darker elements in Figure 2) when performing topology refinement. This is because thinner interconnections immediately introduce topologies with generally much lower stiffness and higher bandgap efficiency. Hence, excluding nodal design variables maintains the focus of Pareto solutions without introducing major changes in the design space. The coarse and refined topologies, therefore, have 136 and 496 independent design variables. Fitness evaluation is performed by the FEM model with the same in-plane discretization of the topology and one element layer through the thickness of the plate.

The optimization algorithm starts with a random binary population of size 200 produced by random mutation of a fully solid material to produce an initial population of likely connected topologies. The morphology of any candidate topology is assessed first and its objective function values are calculated only if the topology includes a valid segment. The valid segment must (i) touch the unit-cell's boundary, and (ii) have appropriate connectivity. The valid segment of coarse topology has to be orthogonally connected, and the valid segment of refined topology is defined to be orthogonally or diagonally connected, in the design variable domain. Apparently, a diagonal connection in the design variable domain of coarse topology introduces undesirable weak hinge connection of topology pixels. This unique valid section only (if any) is passed for evaluation to avoid interrupting modal branches and virtual bandgaps of likely isolated segments (Hedayatrasa et al. 2016b). Elements associated with void regions of topology are removed from the model before analysis, for faster and more accurate fitness evaluation and so no compliant filling material is needed.

The parent pool is generated through a tournament selection, and offspring population is produced by crossover operation and then mutated to retain the diversity of design space. The morphology of corresponding topologies is defined and random objective mutation is performed to firstly fade out the disconnected segments and secondly remove checker-boards (Hedayatrasa et al. 2016b). In the last finishing generations of coarse optimization stage and during refined optimization stage a random edge mutation is exclusively and solely applied to the design variables corresponding to the interfacial pixels of topology. This exclusive edge mutation narrows the search space to the exterior boundaries of topology. This is particularly efficient for the refined design domain, which normally includes many redundant design variables with no effect on the topology (Figure 2(c)). All individuals of offspring and current population are accumulated and sorted based on non-dominated ranking and crowding distance. The new 
generation is filled by the best performing candidates first based on their ranking and if necessary based on their crowding distance (Pratap, Agarwal \& Meyarivan 2002). Finally, the optimized solutions are located on the first non-dominated rank known as Pareto front. 


\subsection{Pareto front and optimized topologies}

From 200 dissimilar topologies achieved in Pareto fronts, optimized topologies TC1-TC8 (with coarse resolution $32 \times 32$ ) and TR1-TR8 (with refined resolution $64 \times 64$ ) are taken and shown in Figure 3(a) and (b), respectively. The topologies are selected such that the extreme topology with maximized RBW (TR1 and TC1) and a number of other intermediate topologies across Pareto front are included.

(Compliant Topology Mode)

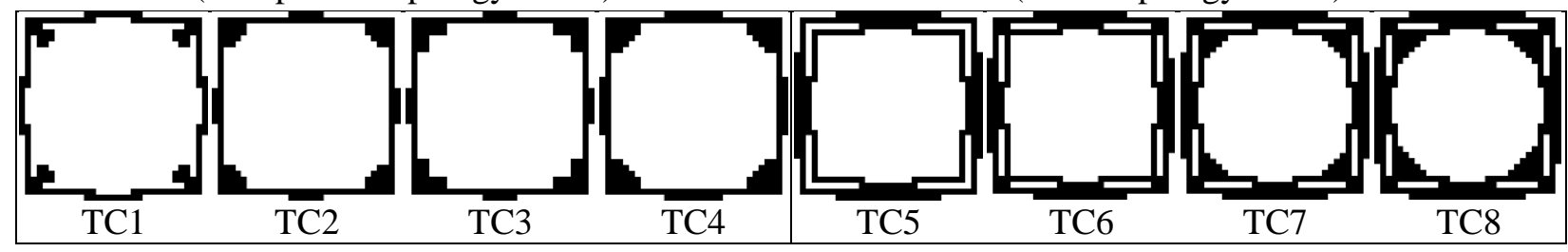

(a) $32 \times 32$

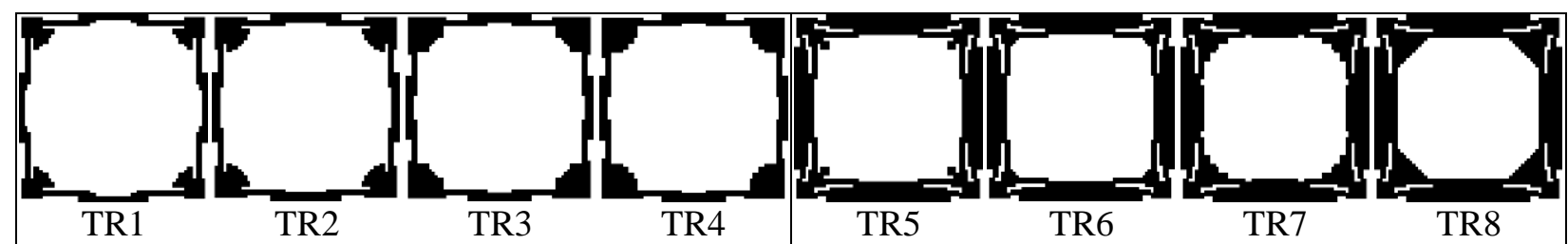

(b) $64 \times 64$

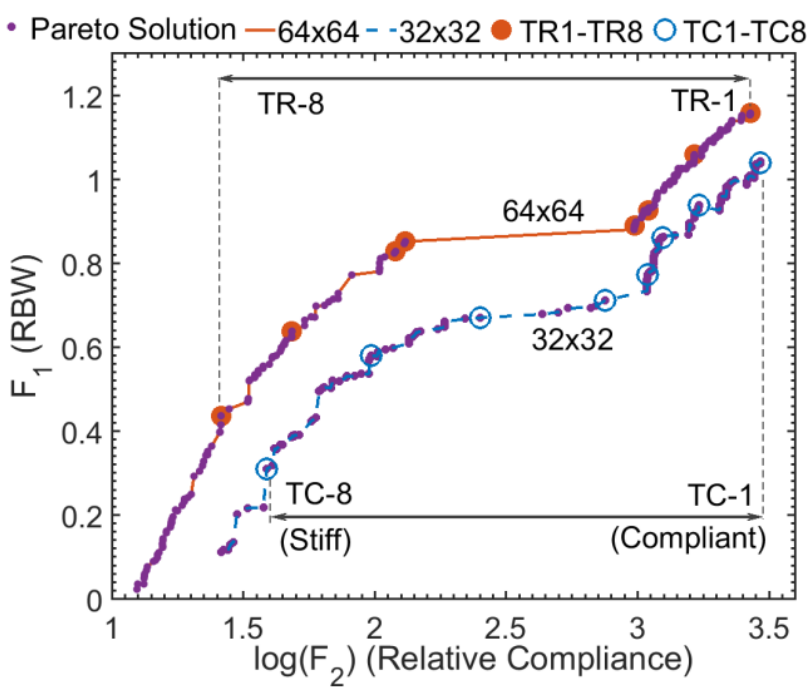

(c)

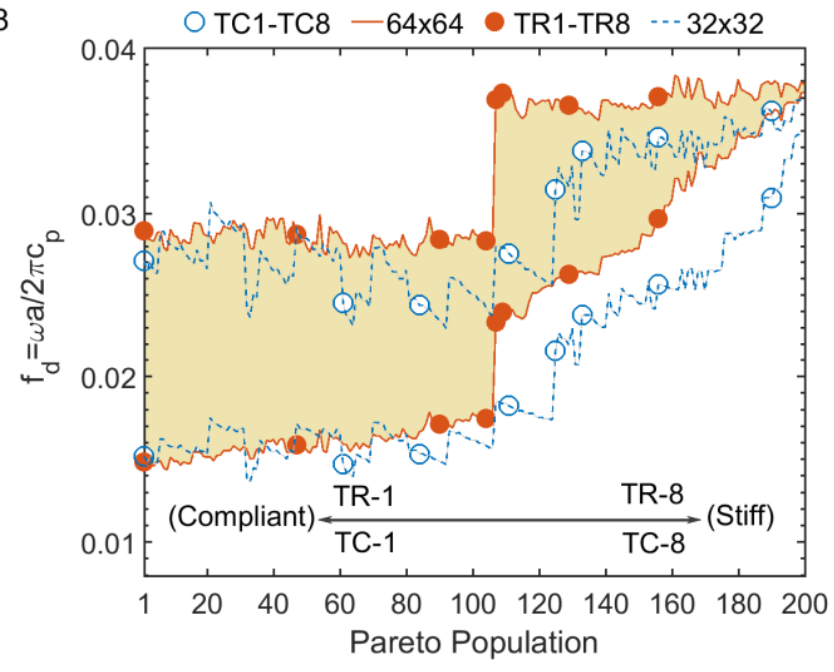

(d)

Figure 3 Selected Pareto topologies of resolution (a) $32 \times 32$ and (b) $64 \times 64$, (c) relevant Pareto fronts, and (d) gradient of bandgap frequency range versus Pareto optimized population of both topology resolutions

The Pareto fronts and relative location of selected topologies are also presented in Figure 3(c). All optimized topologies are evaluated by in-plane FEM resolution of $64 \times 64$, and 8 linear solid element layers are assigned through the thickness. For each Pareto front a set of optimized topologies is achieved in which the bandgap efficiency is degraded by increasing structural stiffness (i.e. reducing compliance). Moreover, by comparison of the two Pareto fronts it is 
evident that the topology refinement significantly enhances the bandgap efficiency and structural stiffness of optimized solutions, and higher RBW is achieved through higher stiffness. The small bold dots in Figure 3(c) show all obtained individual optimized topologies and present the spread of solutions across Pareto Front.

As expected, due to design freedom provided by higher resolution, the refined topologies are more uniformly distributed over the Pareto front compared with the coarse topologies which appear in almost a few clusters. Moreover, a wider spread of topologies are obtained on the high stiffness side through the refined topology. There is a considerable gap among the solutions of refined topology between topologies TR4 and TR5. This gap shows that all alternative intermediate topologies examined during optimization have been dominated by the extreme topologies TR4 and TR5. In fact, transformation of the topology mode from TR4 to TR5 as shown in Figure 3(b) leads to slightly lower bandgap efficiency through much higher stiffness, which naturally pushes intermediate topologies to a lower rank during optimization.

A similar topology transformation is also observed among the optimized coarse topologies (Figure 3(a)) between TC4 and TC5. Of course, the gap between these two coarse topologies is less than the gap between TR4 and TR5 (Figure 3(c)).

The gradient of optimized bandgap frequency range across the Pareto fronts is also shown in Figure 3(d) and bandgap efficiencies of the coarse and refined Pareto population are compared. For generality, dimensionless bandgap frequency $f_{d}=\omega a / 2 \pi c_{p}$ is given in which $c_{p}=\sqrt{E_{s} / \rho_{s}}$ is based on material properties of the solid material. The selected Pareto topologies are also marked by the circle dots to illustrate their relative bandgap frequency range. It is noteworthy that the horizontal axis of Figure 3(d) is just Pareto optimized topology number and does not show the corresponding coarse and refined topologies. The smoother gradient and generally wider bandgaps of refined topologies and the distinct discontinuity in bandgap range of refined topologies are evident. It can be seen how the bandgap frequency range narrows and shifts up by increasing the effective stiffness (from left to right). The stiff topology TR5 has slightly wider bandgap than compliant topology TR4 but at higher frequency range, so providing almost the same RBW.

The structural properties of the various optimized topologies can be further evaluated by comparing their elastic properties. The variation of relative elastic modulus $E_{r}$ and shear modulus $G_{r}$ and effective Poisson's ratio $v_{e}$ across the achieved Pareto population is shown in Figure 4(a) and (b) for topology resolutions $32 \times 32$ and $64 \times 64$, respectively. 


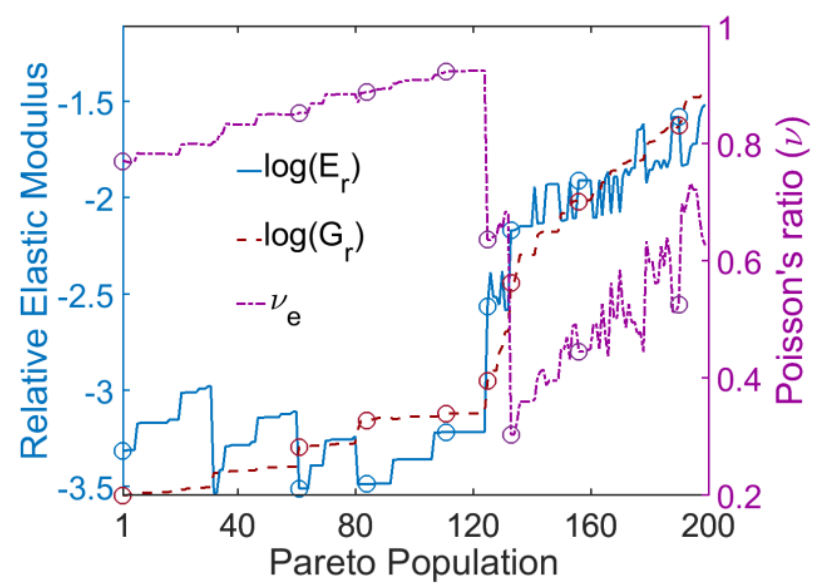

(a)

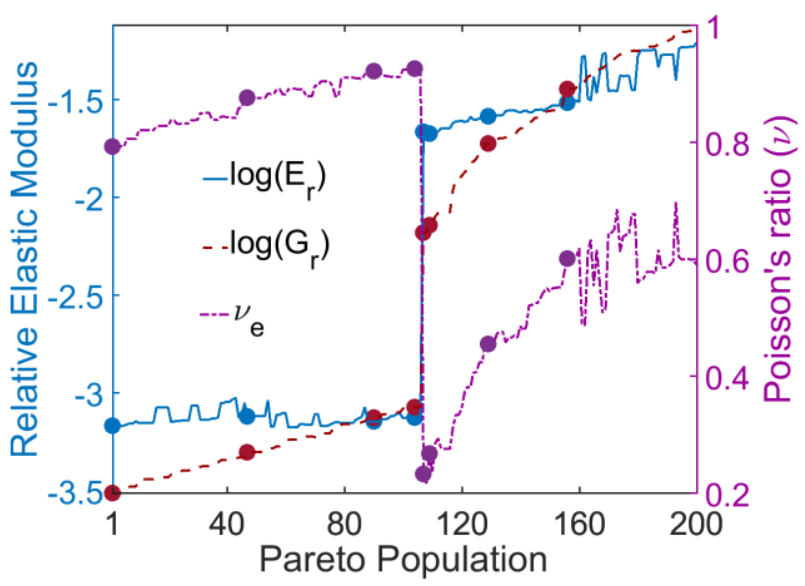

(b)

Figure 4 Gradient of relative elastic and shear modulus $E_{r}$ and $G_{r}$ and effective Poisson's ratio $v_{e}$ versus Pareto population for both topology resolutions (a) $32 \times 32$ and (b) $64 \times 64$, and relative location of selected topologies (Figure 3) marked by circles

The relative elastic and shear modulus is gradually increased through degradation of bandgap efficiency (from left to right) and a sudden jump appears corresponding to the aforementioned gap in the relevant Pareto fronts. The change in the topology mode reduces its effective Poisson's ratio and increases its effective elastic modulus, and according to Eq.5, the first one increases and the second one decreases the normal compliance of the topology. However, according to the Pareto front (Figure 3(c)) the overall stiffness of topology is dominantly increased through this transformation.

The intermediate topologies of Pareto fronts offer great choices for designing functionally graded PhPs in which spatial variation of stiffness is enabled while maintaining an almost uniform bandgap efficiency. Specialized optimizations can be developed to achieve more intermediate topologies e.g. between TR4 and TR5 for this purpose. As an example, subwavelength manipulation of frequencies lower than the bandgap can be achieved through variation of refraction index caused by stiffness gradient, while filtering higher frequencies through bandgap. Also a hierarchical combination of topologies e.g. TR4 and TR7 can produce much wider bandgap from lower band of TR4 to upper band of TR7 (Figure 3(d)), while the stiffness of any individual topology is maintained at its maximized level. The bandgap efficiency of phononic crystals with gradient designs were confirmed by Hussein, Hulbert and Scott (2005) and Hedayatrasa, Abhary and Uddin (2015). 


\section{Experimental evaluation of selected optimized PhPs}

In this section, the results concerning experimental evaluation of produced $\mathrm{PhP}$ topologies are presented and discussed. Aluminum PhPs of a few selected topologies (of both resolutions) are water-jetted and experimentally validated. For each topological resolution, three topologies are chosen for production: compliant topologies (TC4, TR3) and stiff topologies (TC5, TR6) adjacent to the extremes of the discontinuous domain of Pareto fronts, and the stiffest selected topology (TC8, TR8) taken from the high stiffness regime.

Although the refined design domain introduces superior topologies compared with the coarse design domain, it requires a higher precision for manufacturing. Moreover, the refined topologies include narrower void features which require lower perforation beam diameter of the water-jet. To handle this, the coarse topologies and refined topologies are manufactured in an aluminum plate with a thickness of $2.5 \mathrm{~mm}$ and $5 \mathrm{~mm}$, respectively, resulting in unit-cell dimensions of 25 $\mathrm{mm}$ and $50 \mathrm{~mm}$.

The features and the effective elastic properties and bandgap frequencies of the topologies selected for production are summarized in Table 1. To compare with experimental results, these topologies are analyzed by a very fine FEM model using linear solid elements with in-plane mesh resolution of $256 \times 256$ and 20 element layers through the thickness. This higher resolution reduced both calculated modal frequencies and homogenized elastic properties by less than $4 \%$ compared with the resolution of $64 \times 64$ with 8 layers. The dimensionless frequencies of upper and lower limits of bandgap $f_{d, \min }$ and $f_{d, \max }$, respectively, bandgap width $\Delta f_{d}$, midgap frequency $\bar{f}_{d}$ and filling fraction $v_{f}$ are given. The actual upper bandgap frequency $f_{\text {max }}$ and lower bandgap frequency $f_{\min }$ of produced aluminum PhPs are also included.

In order to compare the bandgap efficiency of selected topologies with results of other researchers the RBW is calculated based on both normalized formulations $\Delta f_{d}{ }^{2} / \overline{f_{d}^{2}}$ (Eq.3) and $\Delta f_{d} / \bar{f}_{d}$. The topologies are then reanalyzed by the assumption of aspect ratio $a / h=11$ and Polycarbonate solid background (Halkjær, Sigmund \& Jensen 2006; Bilal \& Hussein 2012), and the relevant normalized bandgaps are included in Table 1 (marked by *). It is obvious that the increased aspect ratio and new material properties slightly reduce the RBW. For the postprocessed optimized topology of Halkjær, Sigmund and Jensen (2006) RBW of $\Delta f_{d} / \bar{f}_{d}=0.16$, and for the optimized topology of Bilal and Hussein (2012) RBW of $\Delta f_{d}^{2} / \overline{f_{d}^{2}}=0.5639$ was reported, confirming the effectiveness of present work in obtaining a wide range of topologies with considerably higher bandgap efficiency. Of course the effective stiffness of topologies 
obtained by referred works are needed to accurately determine their relative location in the Pareto front (Figure 3(c)).

Table 1 The calculated effective elastic properties and bandgap frequencies of coarse and refined Pareto topologies selected for production (Figure 3) and comparison with earlier works

\begin{tabular}{|c|c|c|c|c|c|c|c|c|}
\hline Description & $\begin{array}{l}\text { Com } \\
\text { topo }\end{array}$ & $\begin{array}{l}\text { liant } \\
\text { ogy }\end{array}$ & $\begin{array}{r}\mathrm{St} \\
\text { topc }\end{array}$ & $\begin{array}{l}\text { ff } \\
\text { ogy }\end{array}$ & $\begin{array}{r}\text { High } \mathrm{s} \\
\text { topo }\end{array}$ & $\begin{array}{l}\text { fffness } \\
\text { ogy }\end{array}$ & \begin{tabular}{|c|} 
(Halkjær, \\
Sigmund \& \\
Jensen 2006) \\
\end{tabular} & $\begin{array}{c}\text { Bilal \& } \\
\text { Hussein } \\
\text { 2012) } \\
\end{array}$ \\
\hline Resolution & $32 \times 32$ & $64 \times 64$ & $32 \times 32$ & $64 \times 64$ & $32 \times 32$ & $64 \times 64$ & - & $64 \times 64$ \\
\hline Topology & TC4 & TR3 & TC5 & TR6 & TC8 & TR8 & - & - \\
\hline $\begin{array}{c}a / h \\
(\mathrm{~mm} / \mathrm{mm})\end{array}$ & $25 / 2.5$ & $50 / 5$ & $25 / 2.5$ & $50 / 5$ & $25 / 2.5$ & $50 / 5$ & 11 & 11 \\
\hline$E_{r} \times 10^{3}$ & 0.543 & 0.634 & 2.419 & 18.088 & 23.017 & 26.054 & - & - \\
\hline$G_{r} \times 10^{3}$ & 0.675 & 0.662 & 0.991 & 6.228 & 20.314 & 29.306 & - & - \\
\hline$v_{e}$ & 0.9220 & 0.9198 & 0.6341 & 0.2654 & 0.5252 & 0.5897 & - & - \\
\hline$v_{f}(\%)$ & 22.65 & 23.34 & 28.90 & 36.43 & 37.50 & 43.55 & - & - \\
\hline$f_{d, \min }$ & 0.0178 & 0.0167 & 0.0212 & 0.0232 & 0.0300 & 0.0285 & - & - \\
\hline$f_{d, \max }$ & 0.0273 & 0.0281 & 0.0311 & 0.0369 & 0.0358 & 0.0366 & - & - \\
\hline$\Delta f_{d}$ & 0.0095 & 0.0114 & 0.0099 & 0.0137 & 0.0058 & 0.0081 & - & - \\
\hline $\bar{f}_{d}$ & 0.0226 & 0.0224 & 0.0262 & 0.0300 & 0.0329 & 0.0326 & - & - \\
\hline$\Delta f_{d}^{2} / \overline{f_{d}^{2}}$ & 0.8068 & 0.9560 & 0.7310 & 0.8668 & 0.3499 & 0.4901 & - & - \\
\hline$\Delta f_{d} / \bar{f}_{d}$ & 0.4213 & 0.5089 & 0.3786 & 0.4559 & 0.1763 & 0.2488 & - & - \\
\hline$f_{\min }(\mathrm{kHz})$ & 3.63 & 1.71 & 4.31 & 2.36 & 6.10 & 2.90 & - & - \\
\hline$f_{\max }(\mathrm{kHz})$ & 5.55 & 2.86 & 6.33 & 3.76 & 7.29 & 3.73 & - & - \\
\hline$\Delta f_{d}^{2} / \bar{f}_{d}^{2} *$ & 0.7449 & 0.8998 & 0.6790 & 0.8300 & 0.3195 & 0.4682 & - & 0.5639 \\
\hline$\Delta f_{d} / \bar{f}_{d}^{*}$ & 0.3863 & 0.4753 & 0.3499 & 0.4346 & 0.1608 & 0.2374 & 0.16 & - \\
\hline
\end{tabular}

* Calculated based on aspect ratio $a / h=11$ and Polycarbonate solid material to compare with that of Halkjær, Sigmund and Jensen (2006) and Bilal and Hussein (2012)

\subsection{Validating bandgap of coarse topologies TC4, TC5 and TC8}

For production of coarse topologies, rectangular aluminum plates of $500 \times 400 \mathrm{~mm}$ and thickness $2.5 \mathrm{~mm}$ were water-jetted to make a central lattice array of $10 \times 8 \mathrm{PhP}$ unit-cells with lattice periodicity of $25 \mathrm{~mm}$ as schematically shown in Figure 5(a) for topology TC4. 


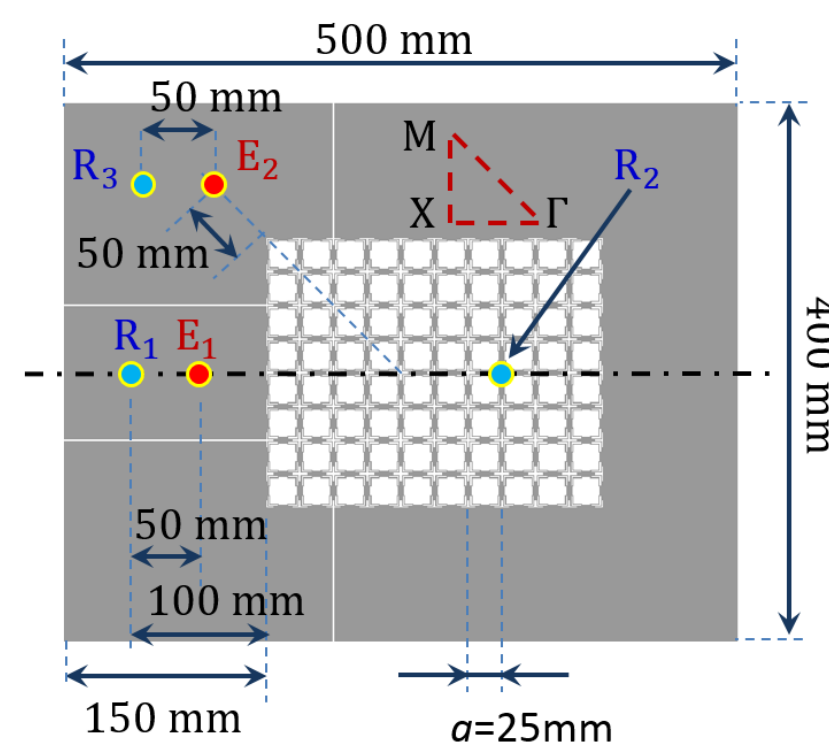

(a)

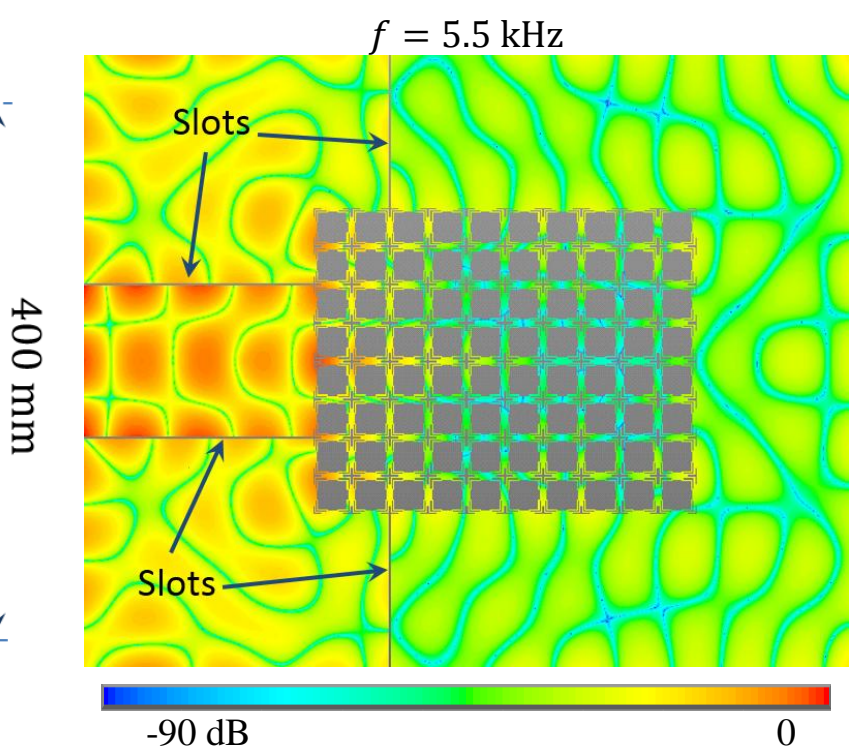

(b)

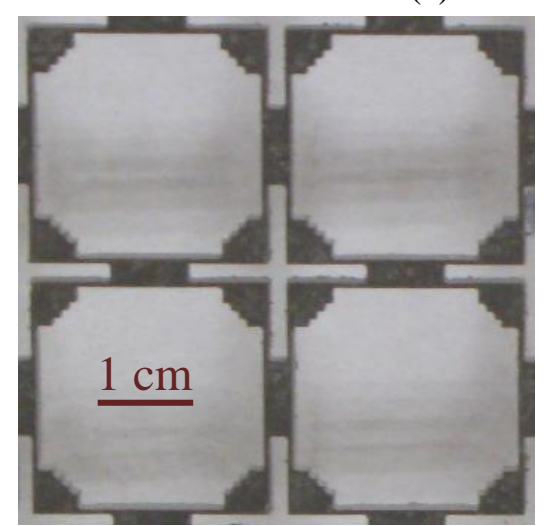

(c)

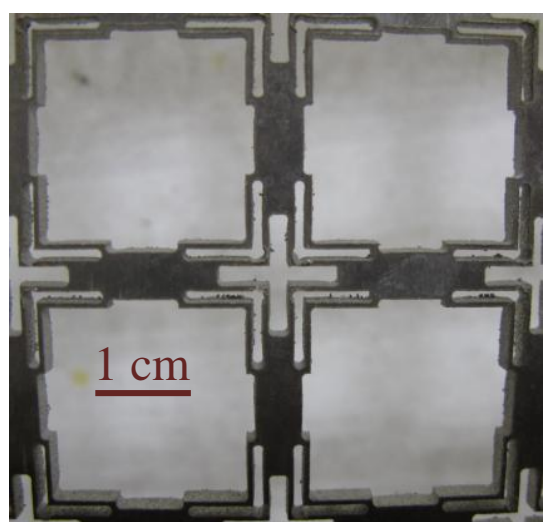

(d)

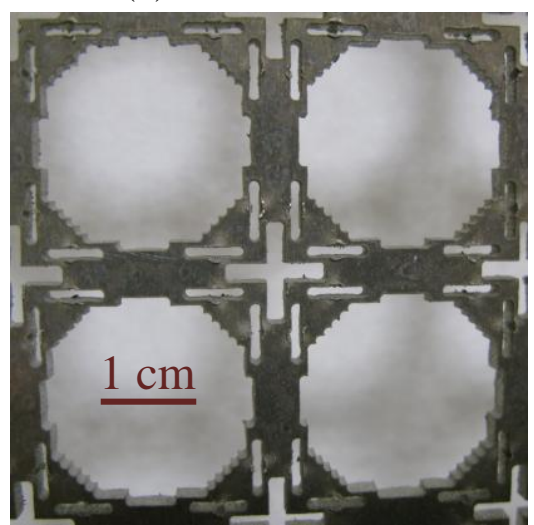

(e)

Figure 5 (Color online). (a) $\mathrm{PhP}$ plate design (shown for topology TC5) and relative location of excitation points $E_{1}$ and $E_{2}$ and measurement points $R_{1}, R_{2}$ and $R_{3}$, and representation of irreducible Brillouin zone ГXM; (b) steady state displacement contour at bandgap frequency $f=5.5 \mathrm{kHz}$ of topology TC4 applied on excitation point $\mathrm{E}_{1}$; and water-jetted PhP of topology (c) TC4, (d) TC5 and (e) TC8 (plate thickness $h=2.5 \mathrm{~mm}$ and unit-cell width $a=25 \mathrm{~mm}$ )

In order to evaluate the bandgap efficiency of the perforated section, the wave is excited at a point on the uniform boundary, either $E_{1}$ or $E_{2}$, and its transmission to different points of the specimen is recorded simultaneously. Slots are cut in the border of the designed $\mathrm{PhP}$ plate in order to isolate the excitation segment from the rest of the plate. It is expected that, in the bandgap frequency range, the wave travelling into the $\mathrm{PhP}$ section is exponentially attenuated and reflected back to the uniform section. The signal measured at $R_{2}$ presents attenuated transmission through $\mathrm{PhP}$ unit-cells, while the transmission of wave through the uniform plate and its resonation by the $\mathrm{PhP}$ section is measured at $\mathrm{R}_{1}$ when exciting $\mathrm{E}_{1}$ and is measured at $\mathrm{R}_{3}$ when exciting $E_{2}$. Although the input signal of $E_{1}\left(\right.$ or $\left.E_{2}\right)$ is known, the signal measured at $R_{1}$ (or $R_{3}$ ) represents the actual resultant spectrum of excitation including the effect of wave scattering at the interface of PhP section and at the boundaries of the Aluminum sample. 
The steady state transmission contour of harmonic excitation at $\mathrm{E}_{1}$ at bandgap frequency 5.5 $\mathrm{kHz}$ for $\mathrm{PhP}$ of topology TC5 is presented in Figure 5(b) in logarithmic scale $\mathrm{dB}$. The exponential wave attenuation throughout the $\mathrm{PhP}$ lattice, wave resonation in the excitation segment and also the function of slots in preventing transmission of waves to the whole border is well illustrated in this contour. The water-jetted topologies TC4, TC5 and TC8 are shown in Figure 5(c), (d) and (e), respectively.

In order to excite and measure flexural waves, circular PZTs are bonded to the top surface of plates with Phenyl Salicylate (Salol). PZTs with a diameter of $20 \mathrm{~mm}$ are used for excitation and PZTs with a diameter of $10 \mathrm{~mm}$ are used for measuring transmitted signals. Bigger exciting PZTs are preferably used to increase the amplitude of excited wave. It is known that the oscillations induced by such circular PZT on one side of the plate dominantly excites the asymmetric wave modes and does not excite symmetric modes to a measurable level within lowfrequency range of interest (Giurgiutiu 2003). The measured signals are recorded with a highresolution 24-bit acquisition card (NI9234) at a sampling frequency of $50 \mathrm{kHz}$ for adequate evaluation of attenuated wave amplitude. The frequency spectra are then calculated using fast Fourier transform (FFT) and are averaged over 20 measurements in order to improve the signalto-noise ratio. All spectra are normalized to the highest transmission amplitude measured at point $\mathrm{R}_{2}$.

A logarithmic frequency sweep excitation voltage $V$ is first applied with amplitude of $V_{0}=100 \mathrm{~V}$, starting from $f_{0}=100 \mathrm{~Hz}$ to $f_{1}=30 \mathrm{kHz}$ over sweep time of $t_{1}=1 \mathrm{~s}$ :

$$
V=V_{0} \cos \left(2 \pi f_{0} \beta^{t} t\right)
$$

so that the coefficient $\beta=\left(f_{1} / f_{0}\right)^{1 / t_{1}}$. This logarithmic sweep enables adequate excitation of low frequencies compared with a linear sweep.

The measured transmission spectra of topology TC5 is presented and evaluated in Figure 6, up to $15 \mathrm{kHz}$. Figure $6(\mathrm{a}-\mathrm{c})$ is concerned with transmission of excitation at $\mathrm{E}_{1}$ to $\mathrm{R}_{1}$ and $\mathrm{R}_{2}$, and Figure $6(d-f)$ is concerned with transmission of excitation at $E_{2}$ to $R_{2}$ and $R_{3}$. The transmission spectrum to the measurement points is shown on the left side. The relative transmission spectrum is also shown on the right hand side which is actually the transmission spectrum of $\mathrm{R}_{2}$ in $\mathrm{PhP}$ section normalized by relevant measured transmission spectrum of uniform section $\left(\mathrm{R}_{1}\right.$ for excitation on $E_{1}$, or $R_{3}$ for excitation on $E_{2}$ ). In fact, the excited spectrum is amplified by resonance of the uniform border and wave reflections from the $\mathrm{PhP}$ section within the bandgap frequency. Hence, the calculated relative transmission gives a better representation of attenuation 
level of any frequency with respect to its relevant excitation level. The calculated modal band structure is also included in the center for verification in which the symmetric and asymmetric modal branches are shown by solid and dashed lines, respectively. The dotted lines highlight the frequency levels corresponding to complete bandgap and also partial bandgap along the Brillouin zone border ГX (Figure 6(b,e)). The spectra shown in Figure 6 evidently confirm the attenuation of transmission up to $-50 \mathrm{~dB}$ within the calculated bandgap frequency range of topology TC5. However, the transmission starts to drop from lower frequency level around $2 \mathrm{kHz}$ at which a partial bandgap opens along Brillouin zone border ГХ.

\section{Pareto Topology TC5 (Tranmission from $\mathrm{E}_{1}$ to $\mathrm{R}_{1}$ and $\mathrm{R}_{2}$ )}

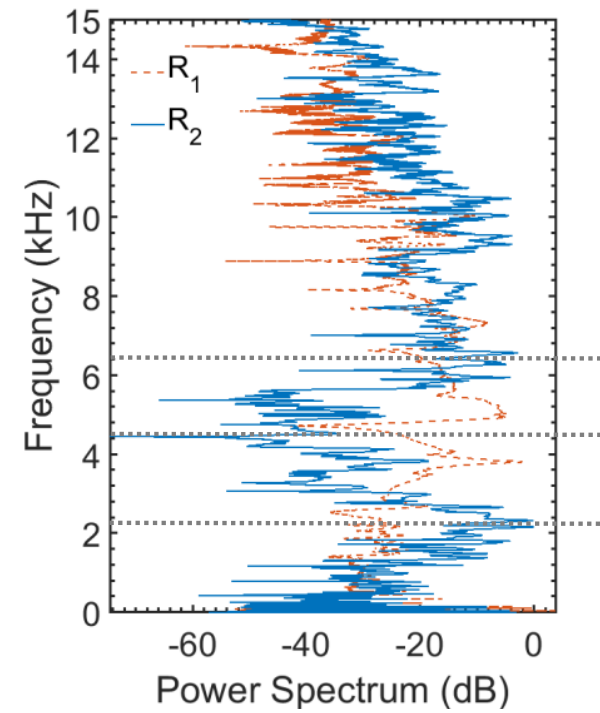

(a)

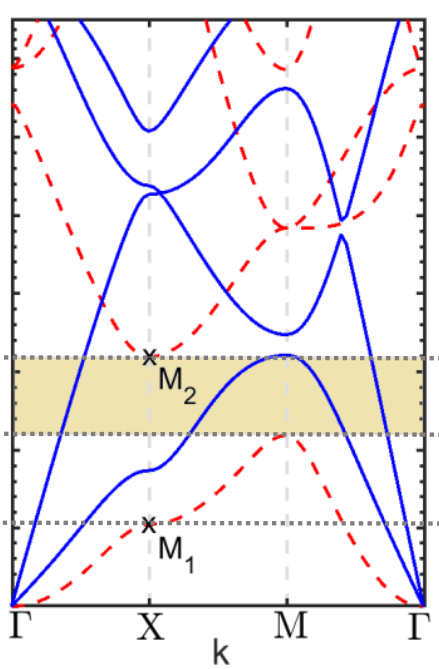

(b)

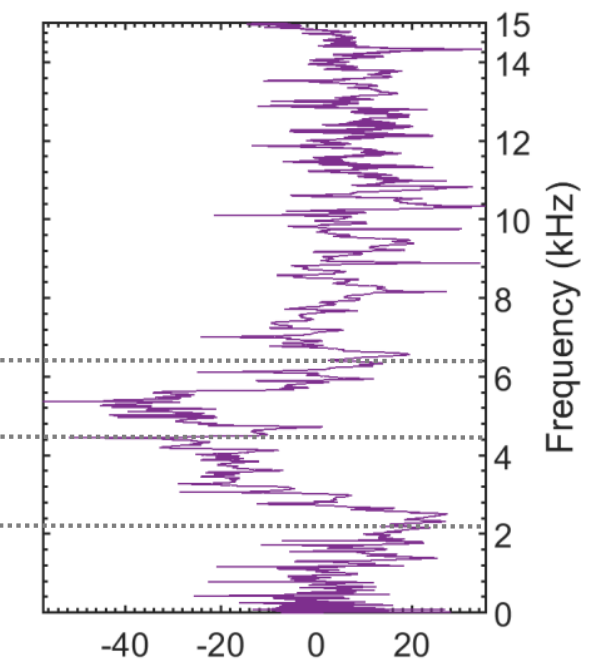

Relative Power Spectrum (dB)

(c)

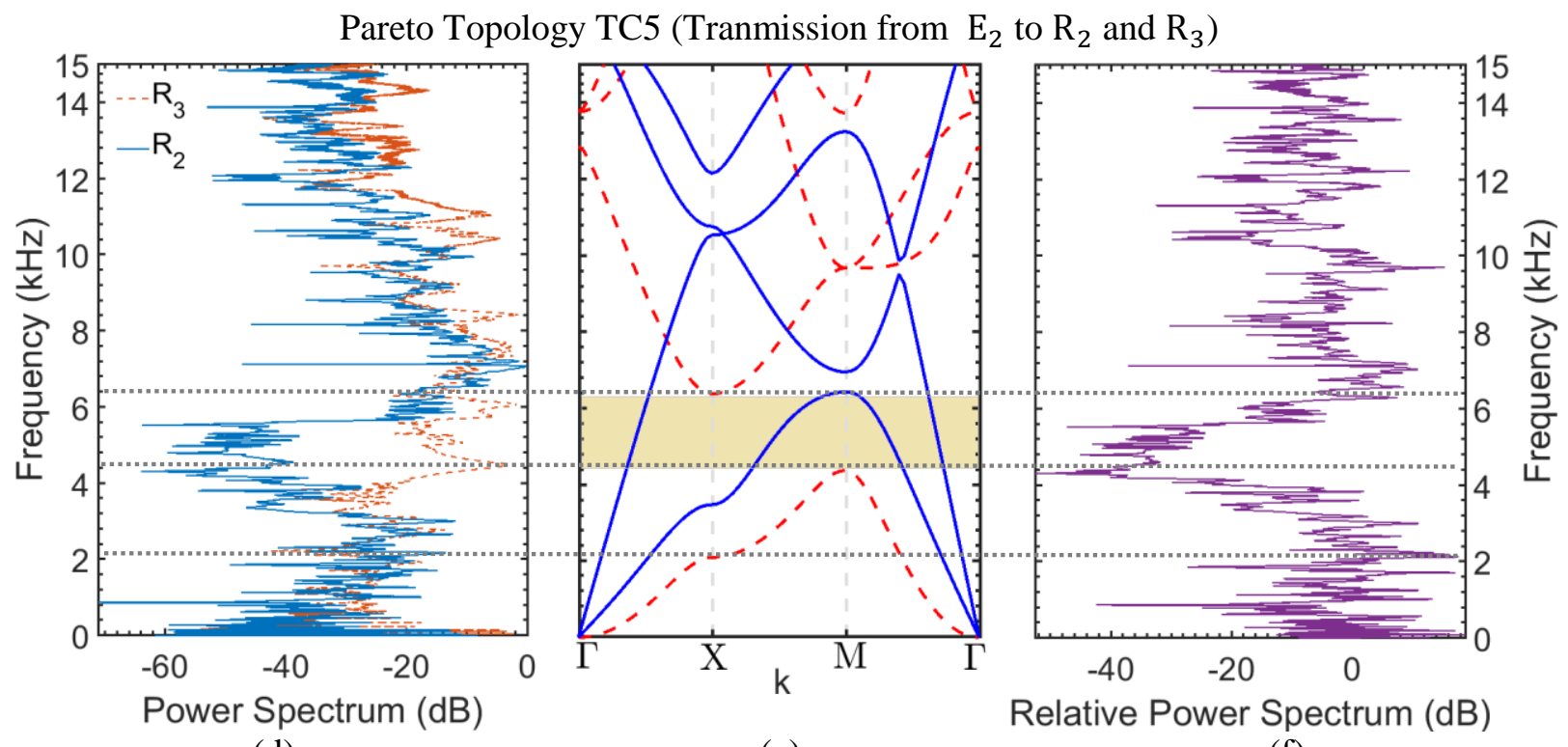

(d)

(e)

(f)

Figure 6 Experimental evaluation of $\mathrm{PhP}$ topology TC5 subjected to frequency sweep excitation: power spectrum of transmission from excitation point (a) $E_{1}$ and (d) $E_{2}$ to measurement points ; (b,e) calculated modal band structure, and relative power spectrum of transmission from excitation point (c) $E_{1}\left(R_{2}\right.$ divided by $\left.R_{1}\right)$, and (f) $E_{2}\left(R_{2}\right.$ divided by $\left.R_{3}\right)$ 
This is more clearly observed in the relative transmission from $\mathrm{E}_{1}$ (Figure 6(c)) which is dominantly orthogonal and along ГX. But in the relative transmission from $\mathrm{E}_{2}$ (Figure 6(f)) the transmission is less affected by this partial bandgap.

In order to reveal the nature of modal branches confining the maximized bandgap, the mode shapes of topology TC5 corresponding to the points $M_{1}$ and $M_{2}$ marked in the relevant modal band structure (Figure 6(b)) are defined and shown in Figure 7. In fact, $M_{1}$ and $M_{2}$ are the first and the second asymmetric modes at Brillouin zone point $\mathrm{X}$ (i.e. $\mathbf{k}=\left[\begin{array}{ll}\pi / a & 0\end{array}\right]$ ) limiting the partial bandgap along the Brillouin zone border $Г X$. The first mode $\mathrm{M}_{1}$ represents a global bending of the lattice uniformly along $\mathrm{y}$-axis, while mode $\mathrm{M}_{2}$ has also oscillations along $\mathrm{y}$-axis. The mode shapes show the wavelength $\lambda$ compared to the unit-cell width $a$ and so the actual wave numbers. In fact, the second mode $\mathrm{M}_{2}$ corresponds to the actual wave vector $\mathbf{k}=$ $\left[\begin{array}{ll}\pi / a & 2 \pi / a\end{array}\right]$ which is folded back to the first Brillouin zone due to periodicity of Bloch-Floquet boundary condition (Eq.2). Thus the bandgap is maximized between fundamental asymmetric Lamb wave mode $\mathrm{A}_{0}$ and its folded modal branch.

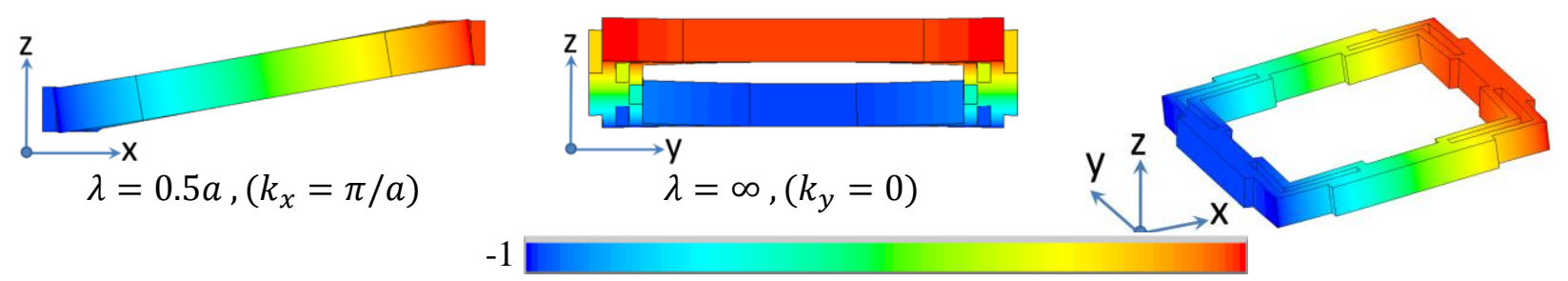

(a) $\mathrm{M}_{1}$ (Figure 6(b))
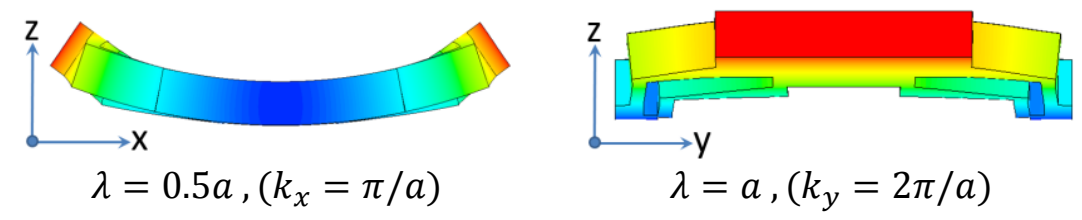

0

(b) $\mathrm{M}_{2}$ (Figure 6(b))

Figure 7 (Color online). Magnified mode shapes of topology TC5 and normalized contour of transversal displacement $w$ along z-axis corresponding to the (a) first and (b) second asymmetric modal branches at Brillouin zone point $\mathrm{X}$ (i.e. $\mathbf{k}=\left[\begin{array}{ll}\pi / a & 0\end{array}\right]$ ) marked in the relevant modal band structure (Figure 6(b)) as $\mathrm{M}_{1}$ and $\mathrm{M}_{2}$

The transmission spectra of frequency sweep excitation on $E_{1}$ for other coarse topologies TC4 and TC8 are also shown in Figure 8 in the same manner. The compliant topology TC4 has a wider complete bandgap attenuating the transmission up to $-60 \mathrm{~dB}$ (Figure $8(\mathrm{c})$ ), and in contrary, the stiff topology TC8 has narrower complete bandgap with less attenuation efficiency up to below $-40 \mathrm{~dB}$ (Figure 8(f)). In both cases, the transmissions start to drop from lower frequency level corresponding to opening the partial bandgap along $\Gamma \mathrm{X}$. Although the complete bandgap of 
TC8 is narrower than TC4, its partial bandgap widths along $Г X$ and $Г \mathrm{M}$ are comparable with TC4, of course at higher frequency range. The transmission spectrum of $R_{1}\left(\right.$ or $\left.R_{3}\right)$ in Figure 6(a,d) and Figure 8(a,d) confirms resonance of the spectrum within the bandgap frequency range due to reflections from $\mathrm{PhP}$ section. Comparison of the modal band structures of the coarse topologies shows how the modal branches are shifted up by increasing stiffness.

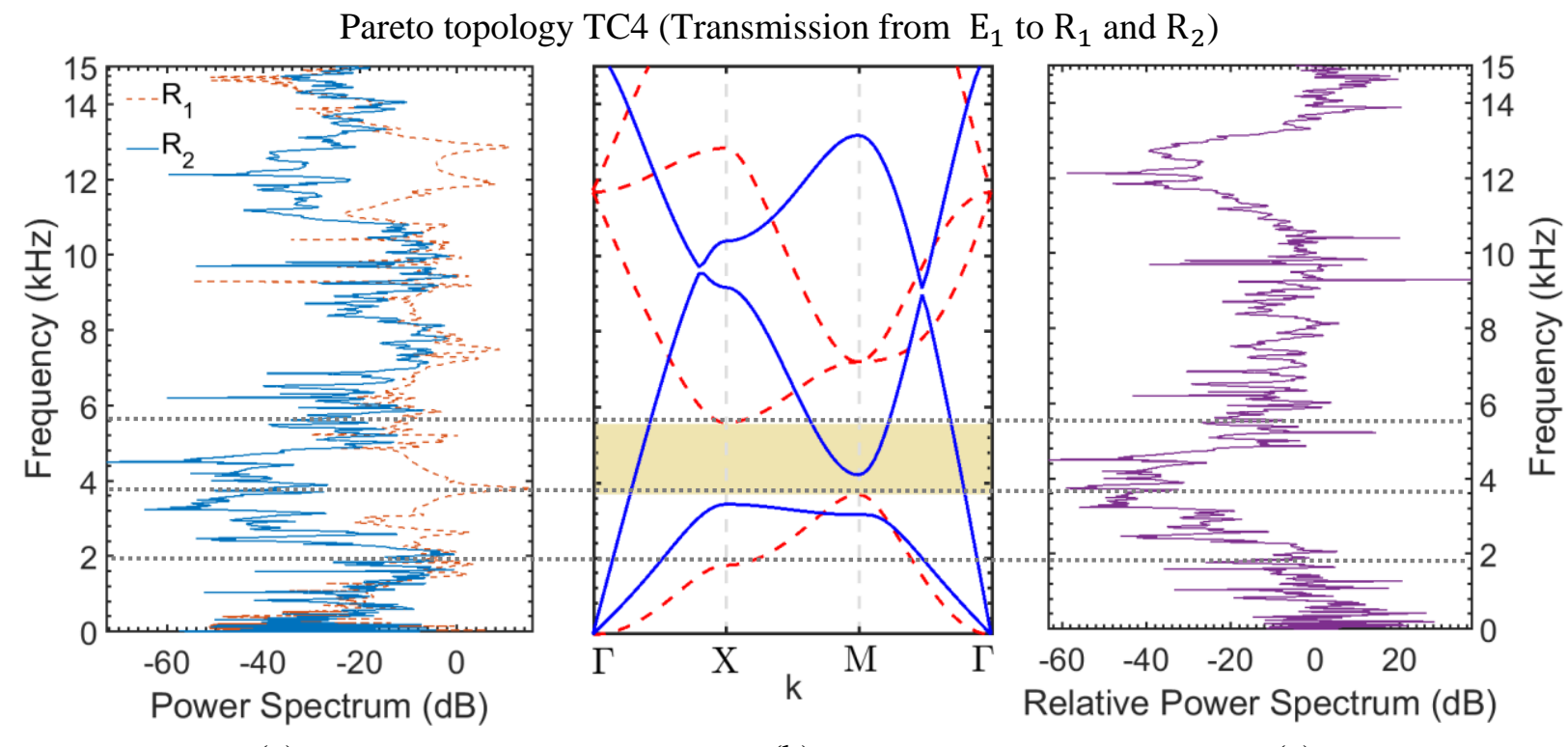

(a)

(b)

(c)

Pareto topology TC8 (Transmission from $\mathrm{E}_{1}$ to $\mathrm{R}_{1}$ and $\mathrm{R}_{2}$ )

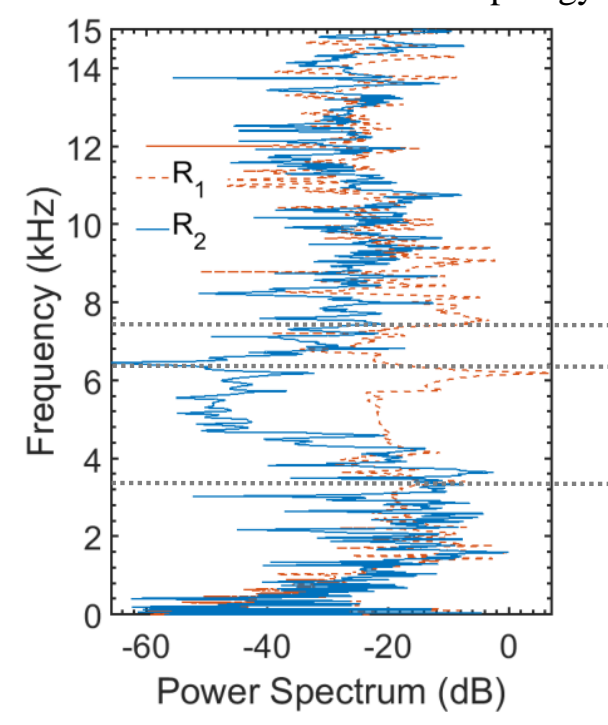

(d)

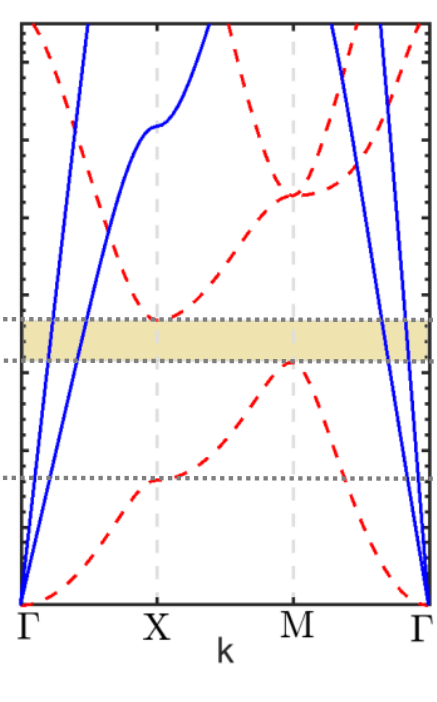

(e)

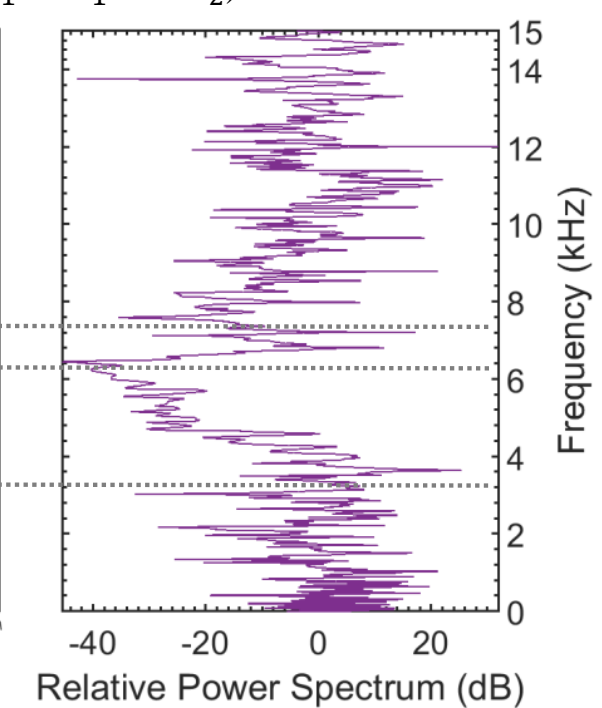

(f)

Figure 8 Experimental evaluation of PhP topologies (a-c) TC4 and (d-f) TC8 subjected to frequency sweep excitation; $(a, d)$ power spectrum transmitted from excitation point $E_{1}$ to measurement points $R_{1}$ and $\mathrm{R}_{2}$; (b,e) calculated modal band structure showing symmetric and asymmetric modes by solid and dashed lines respectively, and (c,f) relative power spectrum of transmission $\left(R_{2}\right.$ divided by $\left.R_{1}\right)$ from excitation point $\mathrm{E}_{1}$ 


\subsection{Validating bandgap of refined topologies TR3, TR6 and TR8}

The $\mathrm{PhP}$ plate with refined topologies, as shown in Figure 9(a) for topology TR3, is designed such that it can be used further for tensile tests and experimental evaluation of elastic properties. An array of $7 \times 7$ unit-cells each $50 \mathrm{~mm}$ wide is perforated in an aluminum plate with thickness $5 \mathrm{~mm}$ and the transmission of Gaussian white noise from excitation point $\mathrm{E}_{3}$ to measurement points $R_{4}$ and $R_{5}$ is determined. The contour of steady state transmission of vibrations excited at $\mathrm{E}_{3}$ with bandgap frequency of $2.5 \mathrm{kHz}$ and its attenuation throughout the designed $\mathrm{PhP}$ is shown in Figure 9(b) for topology TR3. The close view of water-jetted topologies TR3, TR6 and TR8 are shown in Figure 9(c), (d) and (e) respectively.

$$
f=2.5 \mathrm{kHz}
$$
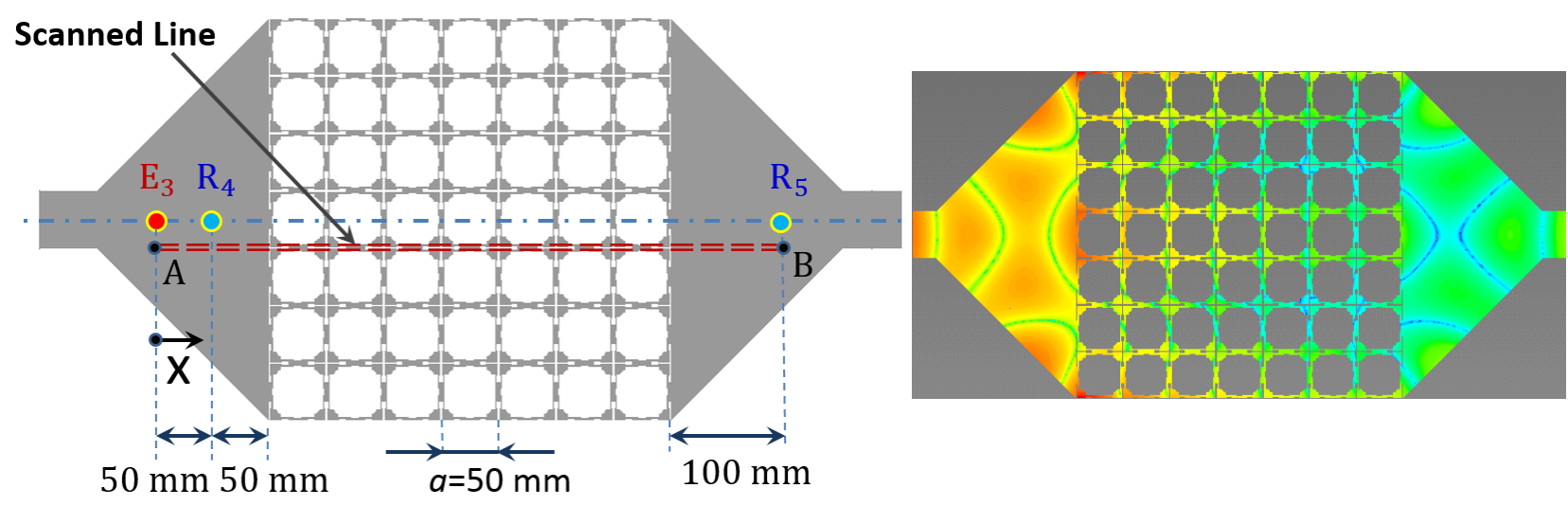

$50 \mathrm{~mm} 50 \mathrm{~mm}$

(a)

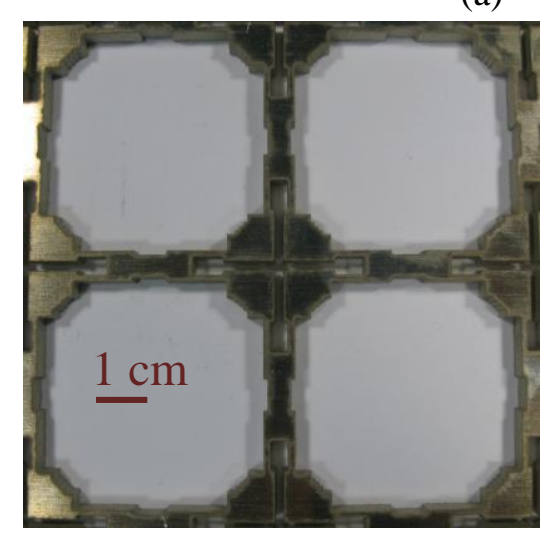

(c)

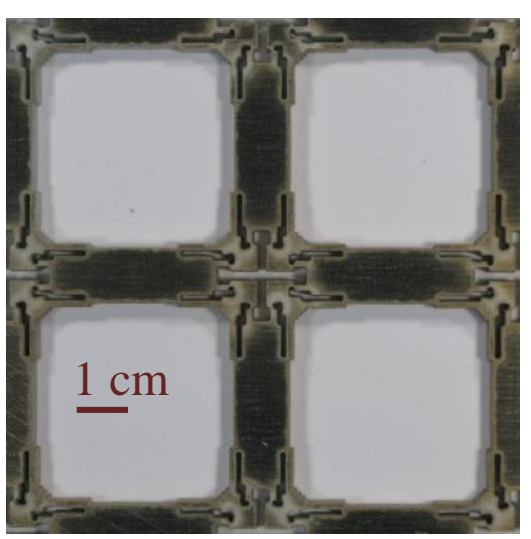

(d)

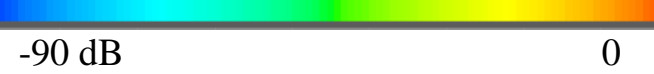

(b)

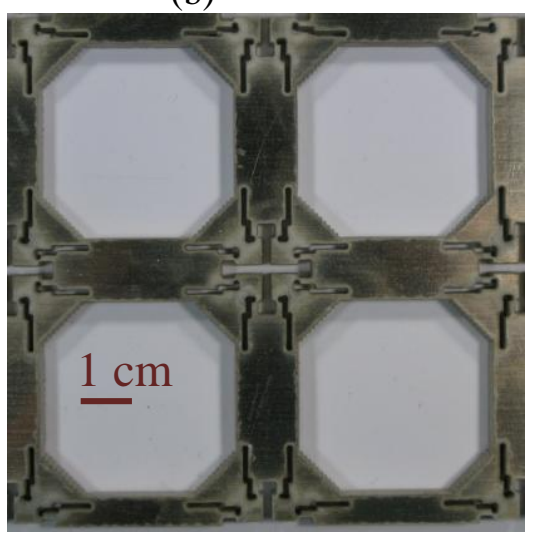

(e)

Figure 9 (Color online). (a) PhP plate design for refined topologies (shown herein for topology TR3) and relative location of excitation point $E_{3}$ and measurement points $R_{4}$ and $R_{5}$, (b) steady state displacement contour at bandgap frequency $f=2.5 \mathrm{kHz}$ of topology TR4 applied on excitation point $\mathrm{E}_{3}$, , and close view of produced topologies (c) TR3, (d) TR6 and (e) TR8; (plate thickness $h=5 \mathrm{~mm}$ and unit-cell width $a=50 \mathrm{~mm}$ ) 
PZTs of diameter $30 \mathrm{~mm}$ are attached to the top surface to excite and measure the signal and the same acquisition card (NI9234) is used. A Gaussian white noise is applied and its transmission spectrum is calculated through FFT over time duration $t=20 \mathrm{~ms}$ as shown in Figure 10, up to $15 \mathrm{kHz}$.

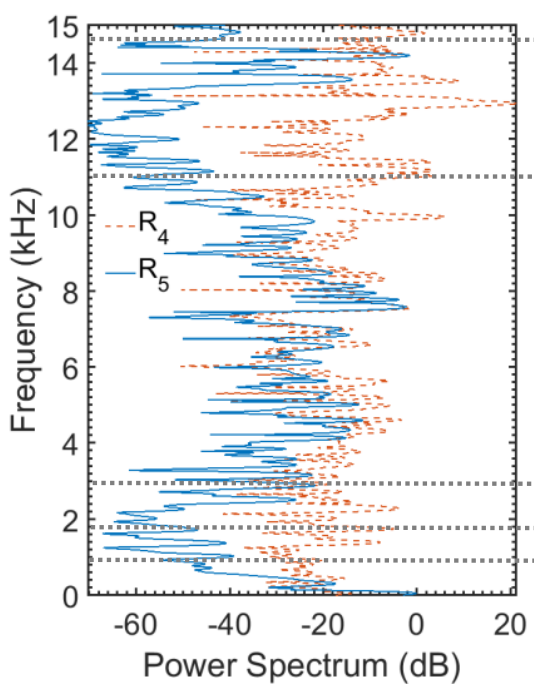

(a)

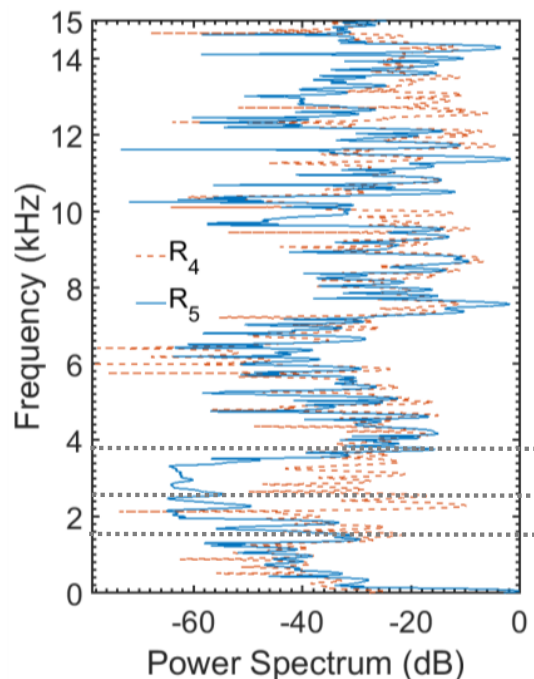

(d)

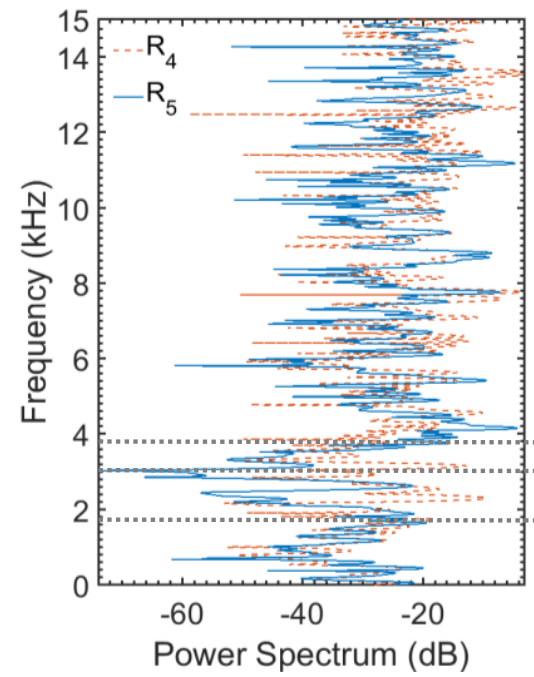

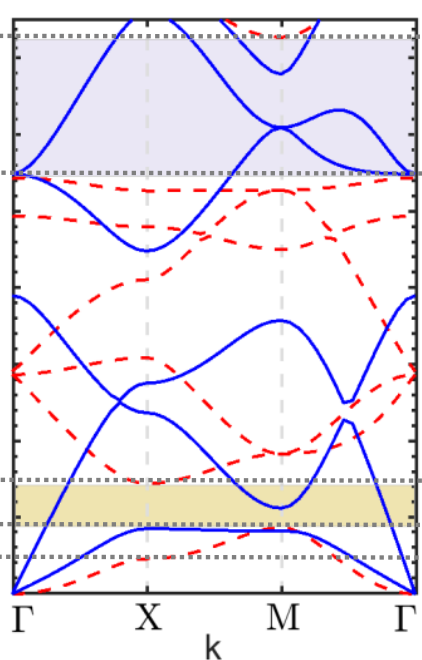

(b) Topology TR3

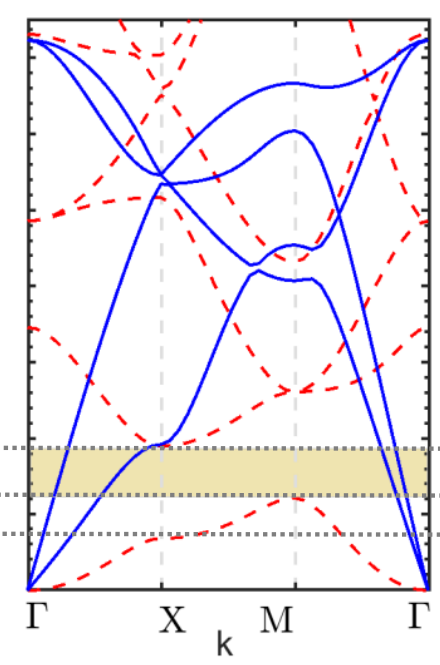

(e) Topology TR6

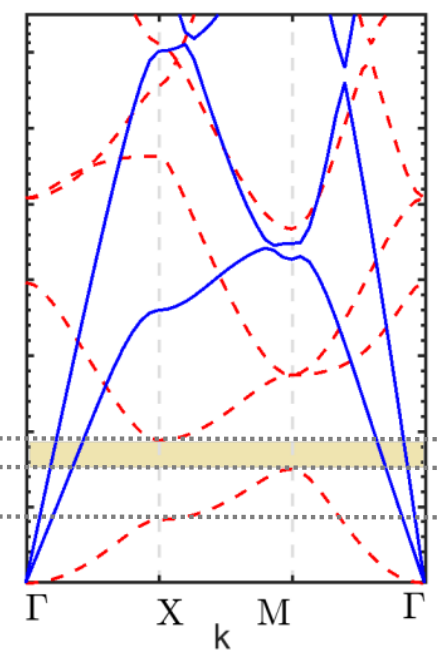

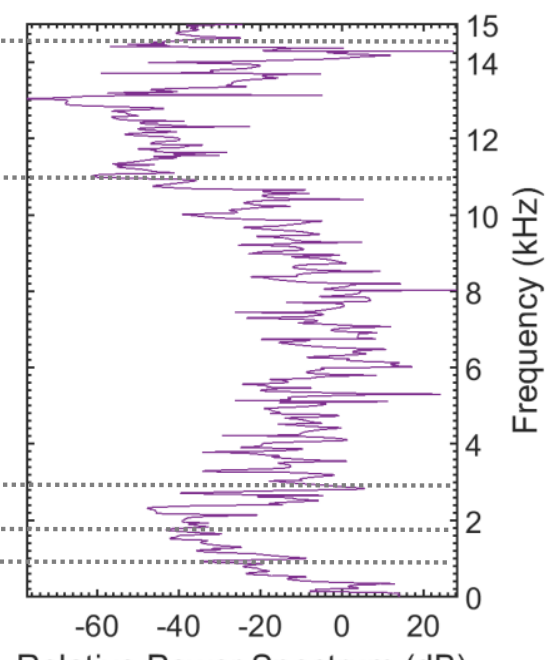

(c)

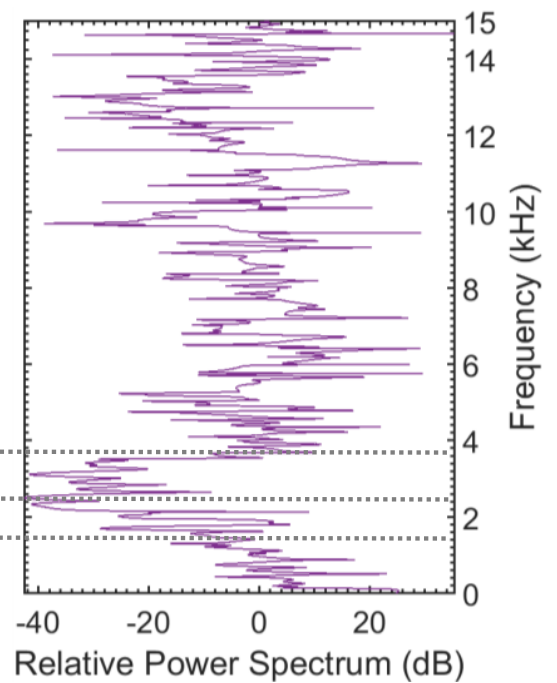

(f)

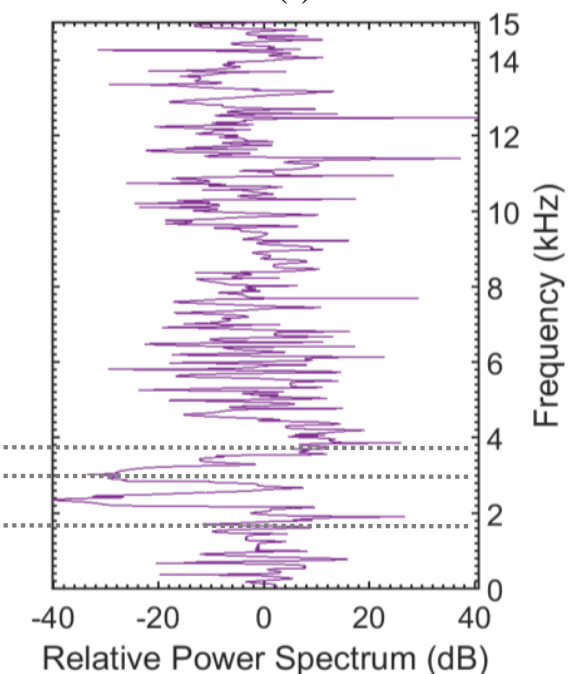


Figure 10 Experimental evaluation of refined PhP topologies (a-c) TR3, (d-f) TR6 and (g-i) TR8 subjected to Gaussian white noise excitation for duration of $t=20 \mathrm{~ms}$ at point $\mathrm{E}_{3}$; $(\mathrm{a}, \mathrm{d}, \mathrm{g})$ power spectrum of transmission to measurement points $\mathrm{R}_{4}$ and $\mathrm{R}_{5}$; (b,e,h) calculated modal band structure showing symmetric and asymmetric modes by solid and dashed lines respectively, and (c,f,i) relative power spectrum of transmission $\left(\mathrm{R}_{5}\right.$ divided by $\left.\mathrm{R}_{4}\right)$

The spectrum of both measurement points which are located on the uniform sections is affected by resonance of the plate. So the applied white noise enables broad band excitation of frequencies and calculation of transmission spectrum over very short time to minimize the effect of resonance of the boundaries. Spectra are normalized to the highest transmission amplitude measured at point $\mathrm{R}_{5}$.

The measured transmission spectra clearly confirm the calculated bandgaps of modal band structures as shown in Figure 10 by a drop in transmission up to $-40 \mathrm{~dB}$. All spectra start to drop at lower frequency level corresponding to opening the partial bandgap of asymmetric wave modes along the Brillouin zone border ГХ. Dominance of this partial bandgap is expected for orthogonal transmission of waves through the PhP from $\mathrm{E}_{3}$ to $\mathrm{R}_{5}$. The compliant topology TR3 has an additional bandgap within the measured frequency range at $11-14.5 \mathrm{kHz}$ with significantly higher attenuation level up to $-80 \mathrm{~dB}$. Comparing the modal band structure of topologies TR6 and TR8 (Figure 10(e \& h)) reveals that their partial bandgap along ГX is almost the same while the complete bandgap of TR8 is considerably narrowed by its lower modal branch.

Furthermore, a short 3 cycle sinusoidal tone-bust with central frequency tuned around the midgap frequency is applied at excitation point $\mathrm{E}_{3}$ and its attenuation through successive $\mathrm{PhP}$ unit-cells is studied by scanning the line AB shown in Figure 9(a) with a laser Doppler vibrometer (LDV) for a duration of $10 \mathrm{~ms}$. The normalized time-frequency spectrum of measured transmission at $x=50 \mathrm{~mm}$ (Figure 9(a)) is calculated by Gabor wavelet transform and shown in Figure 11(a, c \& e) during the first $5 \mathrm{~ms}$. The FFT of transmission to all points scanned over line AB is also calculated and the normalized contour of its variation with respect to the $\mathrm{x}$-axis (Figure 9(a)) is shown in Figure $11(\mathrm{~b}, \mathrm{~d} \& \mathrm{f}$ ); the white sections correspond to perforated parts of line $\mathrm{AB}$. The dotted lines highlight the frequency levels corresponding to complete and partial bandgaps, and the dashed line shows the frequency level of applied excitation.

The Gabor wavelet spectra (Figure 11(a, c \& e)) demonstrate the actual spectrum of excitation and its variation versus time due to wave reflections from the boundary and from porous $\mathrm{PhP}$ section. Initially, the spectrum has the highest magnitude around the applied central 
excitation frequency $2.4 \mathrm{kHz}, 3.0 \mathrm{kHz}$ and $3.5 \mathrm{kHz}$ for topology TR3, TR6 and TR8 (Figure 11(a, c \& e)) respectively. Then the spectrum is dominated by the resonance of upper and lower modes confining the bandgap frequency. With regard to adequate excitation of complete bandgap frequency range, the displacement-frequency FFT contours on the right side of Figure 11 confirm the attenuation of the waves within the bandgap frequency up to $-60 \mathrm{~dB}$ after a few unit-cells. 


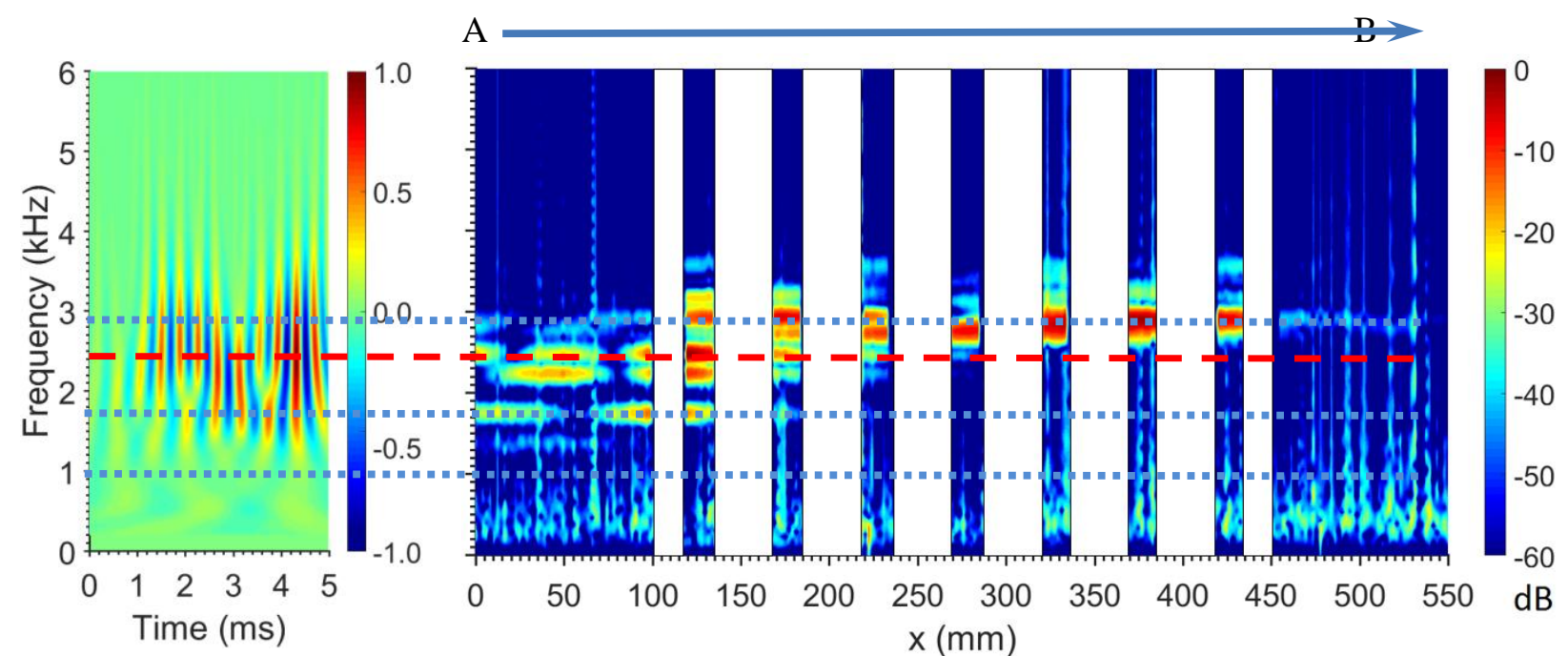

(a) $x=0$

(b) TR3

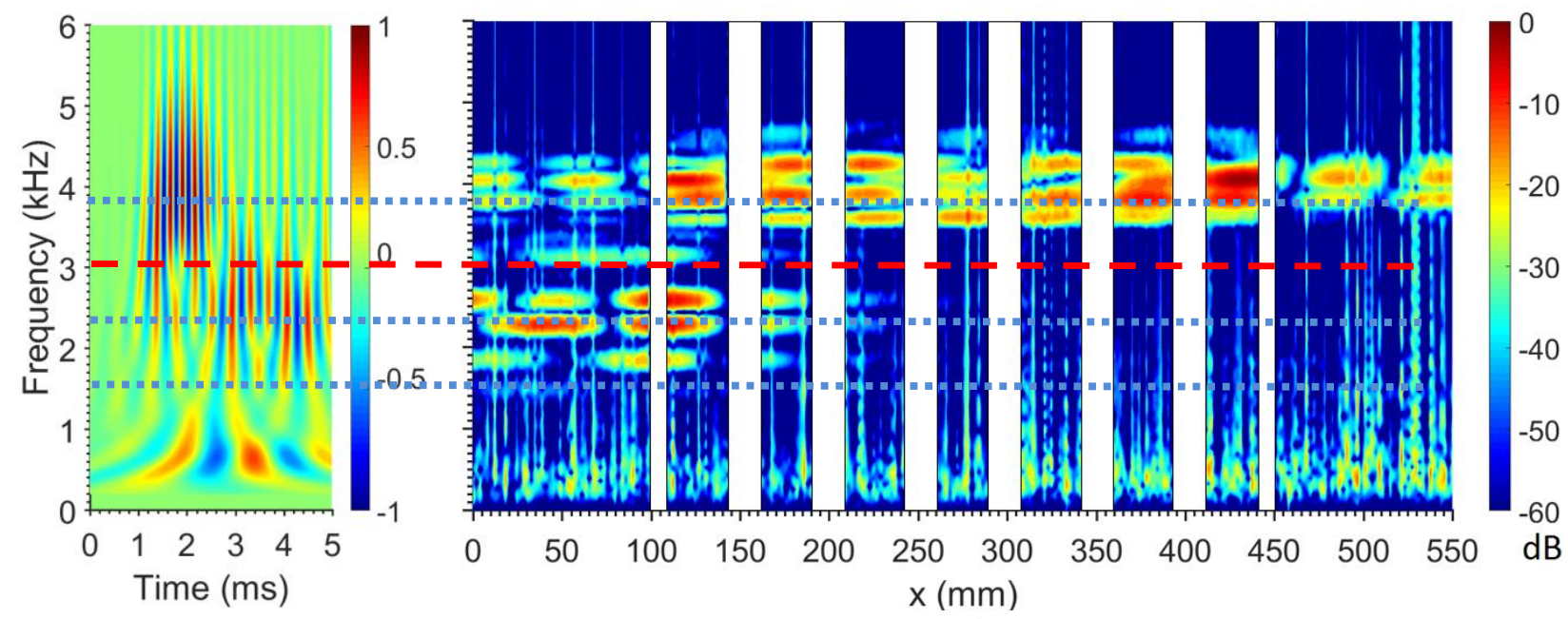

(c) $x=0$

(d) TR6

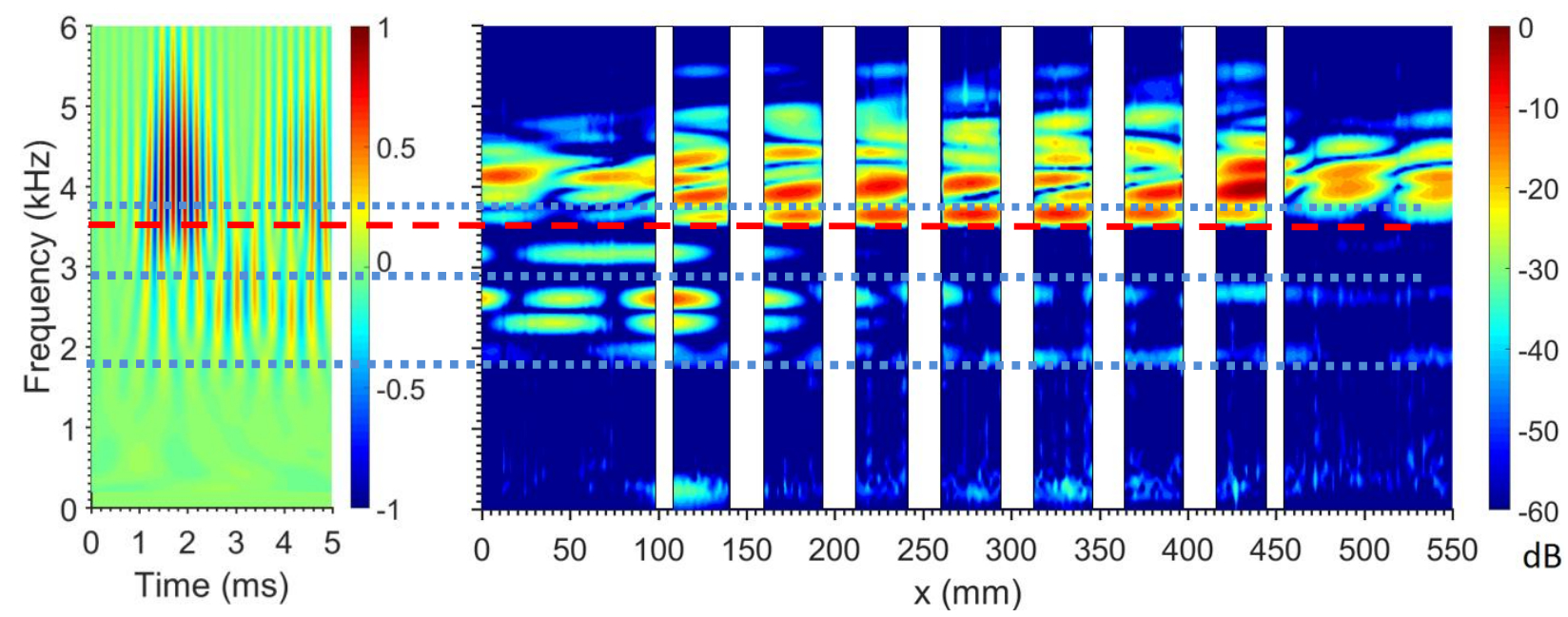

(e) $x=0$

(f) TR8

Figure 11 (Color online). Transmission spectra measured by LDV for 3-cycles sinusoidal tone-burst applied at $\mathrm{E}_{3},(\mathrm{a}, \mathrm{c} \& \mathrm{e})$ normalized Gabor wavelet spectrum of measured excitation at point $\mathrm{A}$ (Figure 9(a)); (b, d \& f) normalized contour of spatial gradient of measured FFT over scanned line AB with length $550 \mathrm{~mm}$ as shown in Figure 9(a); (a,b) Topology TR3 at tone-burst of $2.4 \mathrm{kHz},(\mathrm{c}, \mathrm{d})$ Topology TR6 at $3.0 \mathrm{kHz}$ and (e,f) Topology TR8 at $3.5 \mathrm{kHz}$ 
The measurements of the topologies TR3 and TR6 are affected by an external low-frequency noise source and a high pass filter is applied to the relevant FFT contours to remove the lowlevel noise up to $300 \mathrm{~Hz}$ and clarify the measured excitation. The FFT contours show how the upper and lower modal branches of bandgap are resonated. The upper mode is strongly resonated and the lower mode is decayed after a few unit-cells as it is within the partial bandgap along the Brillouin zone border $\Gamma \mathrm{X}$.

\subsection{Validating stiffness of refined topologies TR3, TR6 and TR8}

The stiffness and effective elastic properties of the optimized topologies, as the second objective of this multiobjective optimization study, are also experimentally evaluated. Produced $\mathrm{PhP}$ specimens of refined topologies TR3, TR6 and TR8, which are designed for this purpose, are stretched in the elastic regime by a tensile test machine as shown in Figure 12(a) and the inplane displacement of the central unit-cell (of $7 \times 7 \mathrm{PhP}$ array) is inspected. The displacement of reference points $\mathrm{M}_{\mathrm{y} 1}, \mathrm{M}_{\mathrm{y} 2}$ and $\mathrm{M}_{\mathrm{x} 1}, \mathrm{M}_{\mathrm{x} 2}$ shown in Figure 12(b) are measured during tensile loading to calculate the longitudinal and transversal strains of the central unit-cell along y-axis and $\mathrm{x}$-axis respectively.

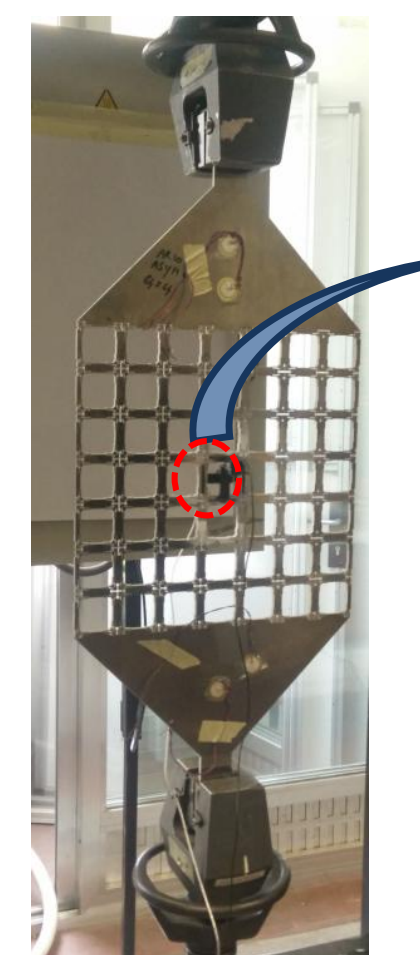

(a)

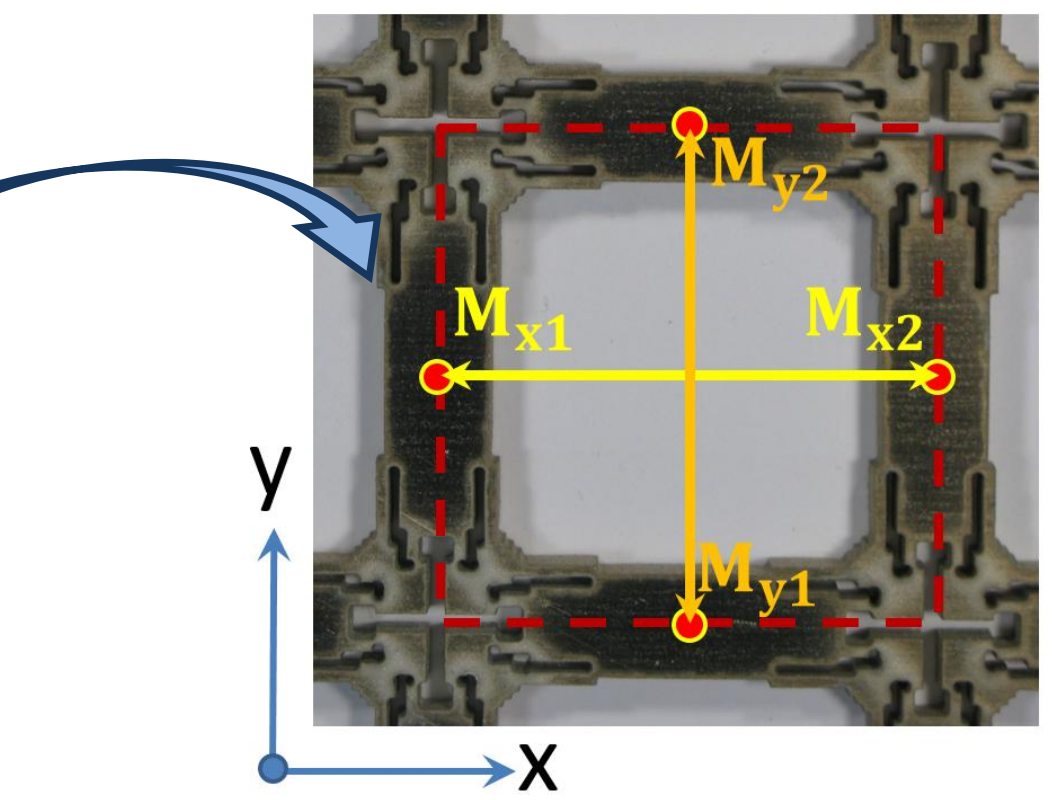

(b)

Figure 12 (a) Tensile test and measuring the displacement field of central unit-cell, (b) measured reference points $\mathrm{M}_{\mathrm{y} 1}, \mathrm{M}_{\mathrm{y} 2}$ and $\mathrm{M}_{\mathrm{x} 1}, \mathrm{M}_{\mathrm{x} 2}$ to calculate the longitudinal and transversal strains of central cell respectively 
Quasi-static tensile tests (force controlled at rate $1 \mathrm{~mm} / \mathrm{min}$ ), are performed on an electromechanical 5800R tensile machine with $10 \mathrm{kN}$ load cell. The maximum loading was taken sufficiently low to remain in the linear elastic region, and displacement over the central unit-cell was measured by (i) longitudinal and transverse extensometers and (ii) stereovision DIC.

In contrast to the use of extensometers, full field strain maps can be obtained by DIC (Sutton, Orteu \& Schreier 2009). This method is based on tracking the geometrical changes in the grey-scale distribution of a speckle pattern which is attached to the specimen surface. A random speckle pattern was applied on the specimen's surface by aerosol spray-paint and is monitored with 2 megapixel 8-bit CCD AVT Stingray F-201 B 1/1.8" cameras throughout the loading event. During quasi-static loading, images with a $1624 \times 1232$ pixel resolution are acquired at a sampling rate of $2 \mathrm{~Hz}$ and are synchronized with the load-displacement signals from the tensile machine. A deformed image is taken at incremental loading steps and compared to the reference image taken prior to loading. This reference image is mapped by a square correlation subset window which is defined by the subset size and step size, being the pixel dimension of one single subset, and the center distance between two adjacent subset windows, respectively. A correlation algorithm computes the displacement of each subset center on the deformed image, estimating the displacement field across the region of interest (ROI). Determination of the specimen's surface displacement field was done using the VIC- $3 \mathrm{D}^{\mathrm{TM}}$ software.

The contours of longitudinal displacement $v$ and transversal displacement $u$ in the central unit-cell of topologies TR3, TR6 and TR8 measured by 3D-DIC are depicted in Figure 13(a-c) respectively. The displacement fields correspond to a tensile load of $250.30 \mathrm{~N}, 756.47 \mathrm{~N}$ and $753.92 \mathrm{~N}$ for TR3, TR6 and TR8 respectively. Higher load level is required for the stiff topologies TR6 and TR8 for reliable measurement of induced displacements, and lower load level is necessary for the complaint topology TR3 to ensure linear-elastic deformation of unitcell. From the measured displacement fields the expected relative compliance of topologies is evident. For a much lower applied load, topology TR3 has much higher longitudinal deformation than TR6 and TR8. Due to symmetry of geometry and boundary conditions of PhP specimens, a uniform displacement field is ideally expected at the central unit-cell. However slight asymmetry is observable for topologies TR6 and TR8 (Figure 13(a-c)) which might be due to rigid body motion of the specimen and also probable misalignment of the tensile load. The almost equal longitudinal extension and lateral contraction of topology TR3 is evident in Figure 13(a). 

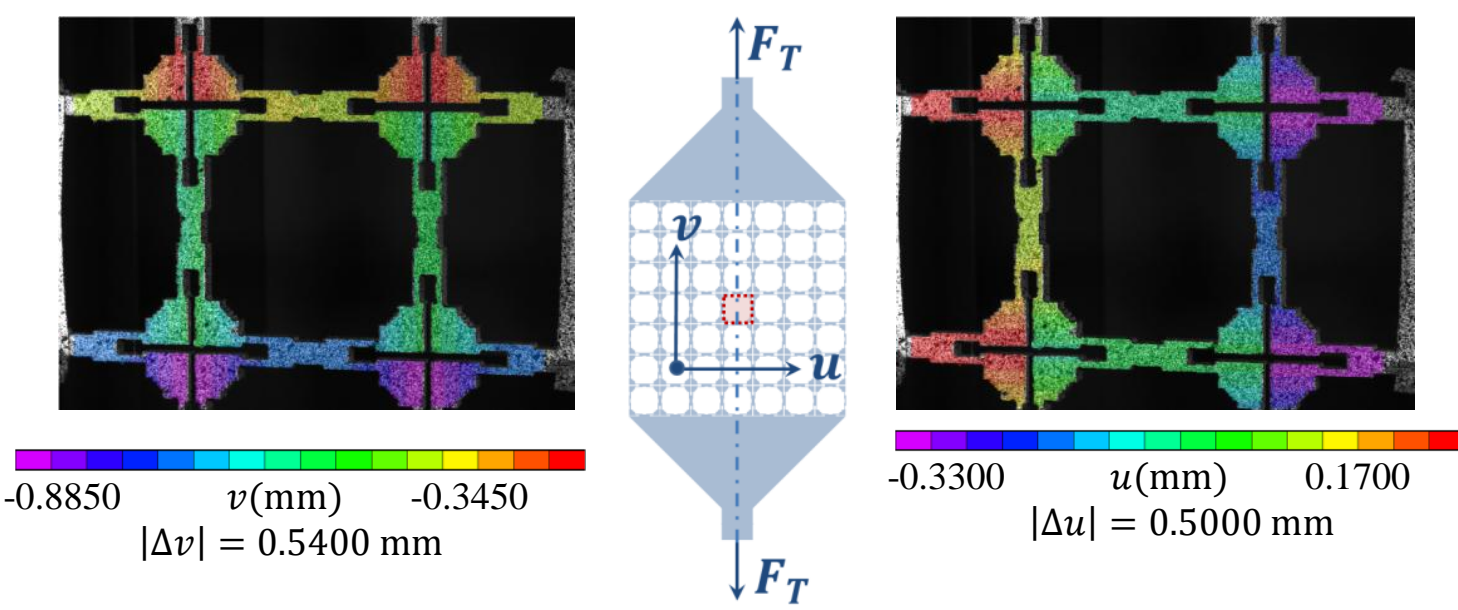

(a) TR3 under $F_{T}=250.30 \mathrm{~N}$
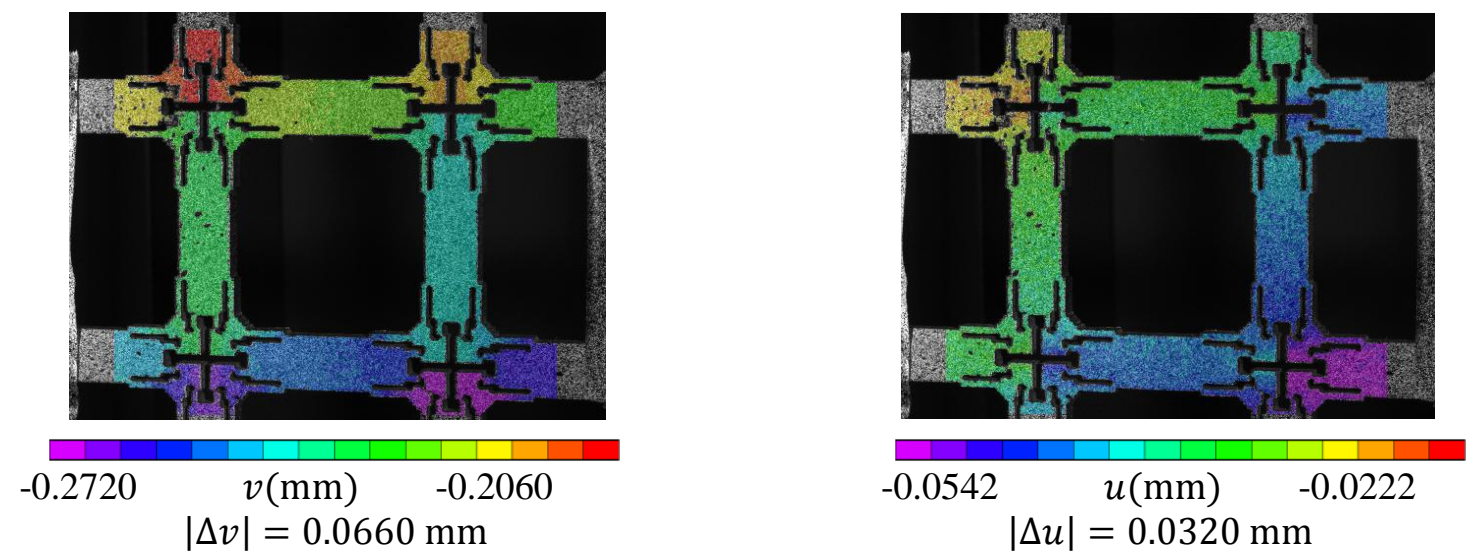

(b) TR6 under $F_{T}=756.47 \mathrm{~N}$
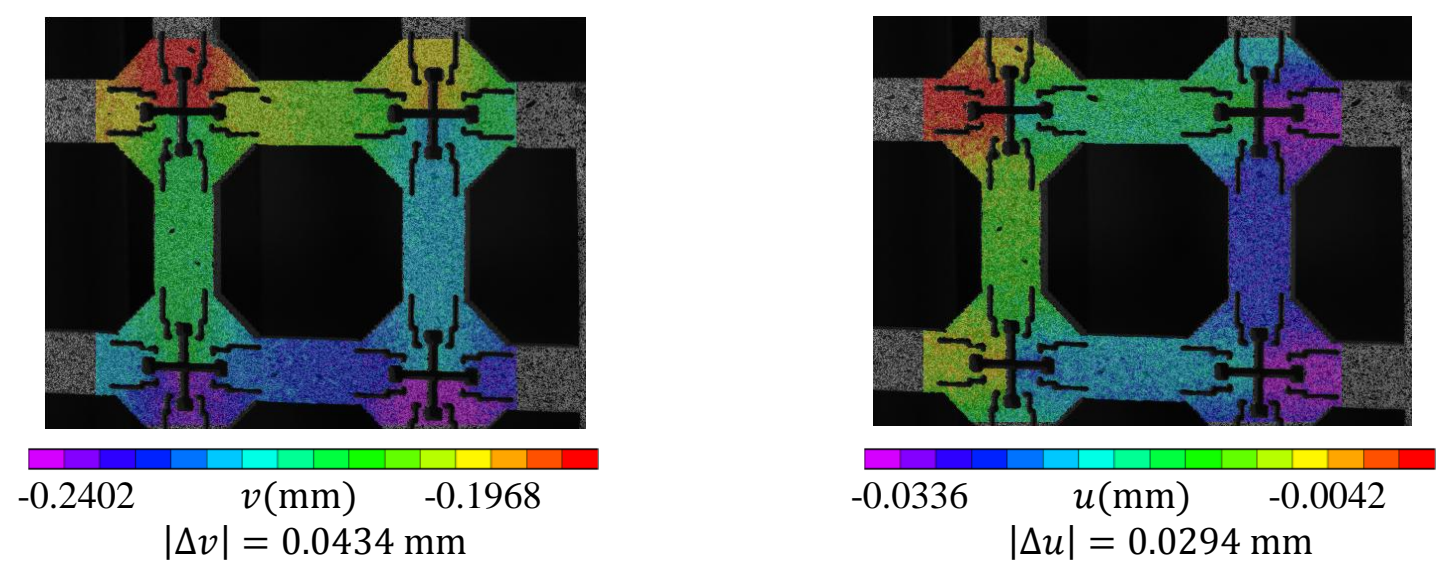

(c) TR8 under $F_{T}=753.92 \mathrm{~N}$

Figure 13 (Color online). Contour of displacement field (longitudinal $v$ and transversal $u$ ) in the central $\mathrm{PhP}$ unit-cell measured by 3D-DIC, (a) TR3 under tensile test load of $F_{T}=250.30 \mathrm{~N}$, (b) TR6 under $F_{T}=756.47 \mathrm{~N}$ and (c) TR8 under $F_{T}=753.92 \mathrm{~N}$

The tensile load is increased in the elastic regime and elastic properties are calculated. By the assumption of a uniform load distribution between the columns of the PhP array, an average stress $\bar{\sigma}_{y}$ and corresponding (secant) elastic modulus $\bar{E}$ are determined from the tensile test measurements. The results concerning compliant topology TR3 are depicted in Figure 14, and all 
measured magnitudes of elastic modulus and Poisson's ratio are summarized in Table 2 and compared with FEM results. Indexes ext, DIC and FEM correspond to tensile test results by extensometers, 3D-DIC and FEM simulation respectively. $E_{\mathrm{e}}$ is the calculated homogenized value based on the relative elastic modulus $E_{\mathrm{r}}$ as given in Table 1. The relevant Poisson's ratio $v$ is also indexed similarly.

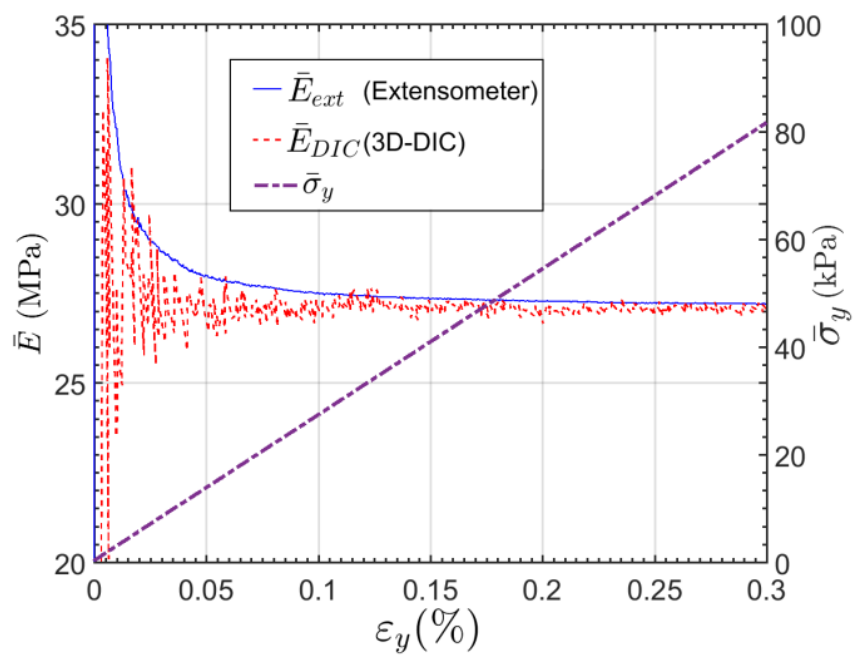

(a)

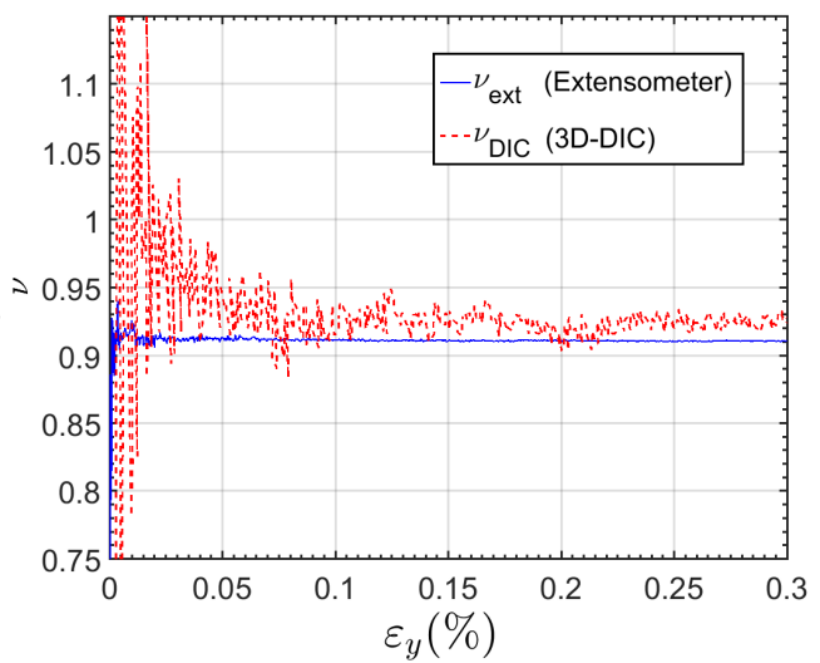

(b)

Figure 14 Tensile test results of topology TR3 measured by 3D-DIC and extensometer in the central PhP unit-cell demonstrating (a) stress $\bar{\sigma}_{y}$ (average over seven $\mathrm{PhP}$ columns) and also corresponding (secant) elastic modulus $\bar{E}$ versus longitudinal strain $\varepsilon_{y}$, and (b) Poisson's ratio versus longitudinal strain $\varepsilon_{y}$

Table 2 Comparison of measured and analyzed magnitudes of elastic modulus $E$ and Poisson's ratio $v$

\begin{tabular}{c|c|cc|cc}
\hline Topology & TR3 & \multicolumn{2}{|c|}{ TR6 } & \multicolumn{2}{c}{ TR8 } \\
& Absolute & Absolute & $\begin{array}{c}\text { Relative to } \\
\text { TR3 }\end{array}$ & Absolute & $\begin{array}{c}\text { Relative to } \\
\text { TR3 }\end{array}$ \\
\hline $\bar{E}_{\text {ext }}$ & $27.25 \pm 0.03(\mathrm{MPa})$ & $905.0 \pm 5.1(\mathrm{MPa})$ & 33.21 & $1288.8 \pm 3.3(\mathrm{MPa})$ & 47.30 \\
$\bar{E}_{\mathrm{DIC}}$ & $26.96 \pm 0.16(\mathrm{MPa})$ & $909.1 \pm 35.3(\mathrm{MPa})$ & 33.72 & $1436.7 \pm 66.1(\mathrm{MPa})$ & 53.29 \\
$\bar{E}_{\mathrm{FEM}}$ & $33.88(\mathrm{MPa})$ & $1129.17(\mathrm{MPa})$ & 33.33 & $1468.19(\mathrm{MPa})$ & 43.34 \\
$E_{\mathrm{e}}$ & $44.38(\mathrm{MPa})$ & $1266.16(\mathrm{MPa})$ & 28.53 & $1823.78(\mathrm{MPa})$ & 41.09 \\
\hline$v_{\text {ext }}$ & $0.9109 \pm 0.0003$ & $0.2567 \pm 0.0011$ & - & $0.6361 \pm 0.0012$ & - \\
$v_{\mathrm{DIC}}$ & $0.9254 \pm 0.0054$ & $0.2693 \pm 0.0498$ & - & $0.6814 \pm 0.0567$ & - \\
$v_{\mathrm{FEM}}$ & 0.9101 & 0.2555 & - & 0.6175 & - \\
$v_{\mathrm{e}}$ & 0.9198 & 0.2654 & - & 0.5897 & - \\
\hline
\end{tabular}

The stress-strain curve depicted in Figure 14(a) presents the linear elastic response of $\mathrm{PhP}$ specimen, in which the results from both extensometer and 3D-DIC measurements coincide. More details are revealed in the corresponding elastic modulus (Figure 14(a)) and Poisson's ratio 
(Figure 14(b)) showing relatively large deviations up to around $\varepsilon_{y}=0.1 \%$ due to the inevitable error in measuring very small displacements below the accuracy limit of extensometers and 3DDIC. After this unsteady stage, the elastic modulus and Poisson's ratio measured by extensometer and 3D-DIC flatten, converge and have a good agreement.

According to the results given in Table 2, it can be argued that:

- The measured Poisson's ratios and those from FEM analysis are in very good agreement with the homogenized ones.

- For all topologies the homogenized values of the elastic modulus $E_{\mathrm{e}}$ are larger than those corresponding to the tensile test $\bar{E}$. This discrepancy is mainly due to the assumption of uniform load distribution between PhP columns when calculating $\bar{E}$ which is compromised by the finite nature of the $\mathrm{PhP}$ specimen (see Appendix A).

- The experimentally defined elastic modulus $\bar{E}_{\text {ext }}$ and $\bar{E}_{\text {DIC }}$ are in relatively good agreement and both are less than $\bar{E}_{\mathrm{FEM}}$, except for TR8 of which $\bar{E}_{\mathrm{DIC}}$ is very close to $\bar{E}_{\mathrm{FEM}}$. The discrepancy of experimental results (i.e. $\bar{E}_{\text {ext }} \& \bar{E}_{\text {DIC }}$ ) with results from FEM simulation of tensile test (i.e. $\bar{E}_{\mathrm{FEM}}$ ) can be due to: (i) measurement errors, (ii) the deviation of waterjetted unit-cell topologies compared with obtained optimized topology (we noted for example that the water-jet beam is not perfectly straight, resulting in slightly different sections at top and bottom), (iii) the difference between actual elastic properties of the aluminum plate with those considered for modelling and (iv) approximations inherent to FEM analysis.

However, it is evidently shown that the relative elastic modulus of produced stiff topology TR6 with respect to TR3 is around 33 (Table 2) for both experimental and FEM results, while their almost equal RBW was also experimentally observed in Section 3.2 (Figure 10(a-f)). Moreover, the relative elastic modulus of produced TR8 with respect to that of produced TR3 is 47.30 and 53.29 based on extensometer and 3D-DIC measurements, respectively, which both are higher than that of FEM calculations 43.34. The bandgap efficiency of the produced PhP of TR8 was also confirmed compared with its calculated RBW (Figure 10(g-i)). Consequently, the relative effective stiffness and bandgap efficiency of topologies are experimentally validated as expected from the obtained Pareto front (Figure 3(c)). 


\section{Conclusion}

Porous phononic crystals, manufactured by through-thickness perforation of a uniform plate, were optimized for maximized bandgap of flexural guided wave modes and maximized stiffness. Aluminum specimens of selected topologies were water-jetted and their calculated relative bandgap efficiency and effective stiffness were experimentally confirmed.

The lowest bandgap between the first couple of modal branches (i.e. fundamental asymmetric Lamb mode $A_{0}$ and its folded branch) was maximized. Coarse topologies of resolution $32 \times 32$ were first achieved. Then the resolution was refined to $64 \times 64$ while constraining the solid feature sizes through a projection approach. The Pareto fronts of relevant multiobjective optimizations were presented and it was shown how the topology refinement enhances the performance of optimized topologies and how bandgap efficiency of topology is degraded by increasing its effective stiffness. The Pareto topologies were compared and distinct compliant and stiff topology modes were realized providing almost the same bandgap efficiency.

The transmission spectrum of frequency sweep and random white noise excitations through the water-jetted specimens indicated a considerable wave attenuation within calculated bandgap frequency range and extended down to the frequency level concerning relevant partial bandgap. Scanning the wave transmission throughout the specimens by LDV clearly demonstrated the attenuation of excited waves within the bandgap frequency and resonance of bandgap side bands. The stiffness of selected specimens was also evaluated through tensile tests and the deformation of the central unit-cell of the phononic lattice was inspected by extensometer and 3D-DIC. The elastic modulus and Poisson's ratio of the various topologies were experimentally determined, showing good agreement with FEM results. The relative stiffness of topologies with respect to their bandgap efficiency as expected from Pareto fronts was confirmed.

The variety of topologies obtained between stiff and compliant extremes of achieved Pareto fronts offers great choices for designing graded phononic crystal plates in which spatial variation of stiffness is enabled while maintaining an almost uniform bandgap efficiency. Hierarchical arrangement of selected topologies also offers creation of very wide bandgaps while maintaining the overall stiffness at the maximized level. If a unidirectional bandgap efficiency is desired then the topologies providing higher flexibility as partial bandgaps were shown to be much less sensitive to the stiffness. This potentially motivates a multiscale optimization study to take the advantage of these capabilities in achieving additional functionality at the structure scale. 


\section{Acknowledgements}

S. Hedayatrasa acknowledges the University of South Australia Postgraduate Research Award (USAPA) and student mobility international travel grant.

M. Kersemans acknowledges the financial support of Bijzonder OnderzoeksFonds (BOF) Grant No. BOF.PDO.2015.0028.01.

J.K. Guest acknowledges the support of the U.S. National Science Foundation under Grant No. 1400394.

The authors would also like to thank Cléante Dierickx (Odisee) for manufacturing the different PhPs through water-jetting.

\section{Appendix A.}

In order to realize the deformation and loading conditions of the central unit-cell, the tensile test is simulated by ANSYS APDL (ANSYS® Academic Research, Release 16). An equal tensile load of $F_{T}=70 \mathrm{~N}$ is applied to $\mathrm{PhP}$ specimens of experimentally verified topologies and resultant stress intensities and the edge loads of central unit-cell are defined as shown in Figure A.1.

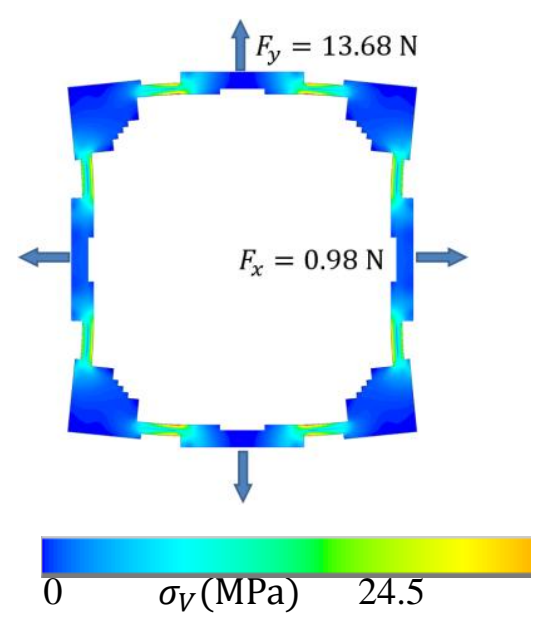

(a) TR3

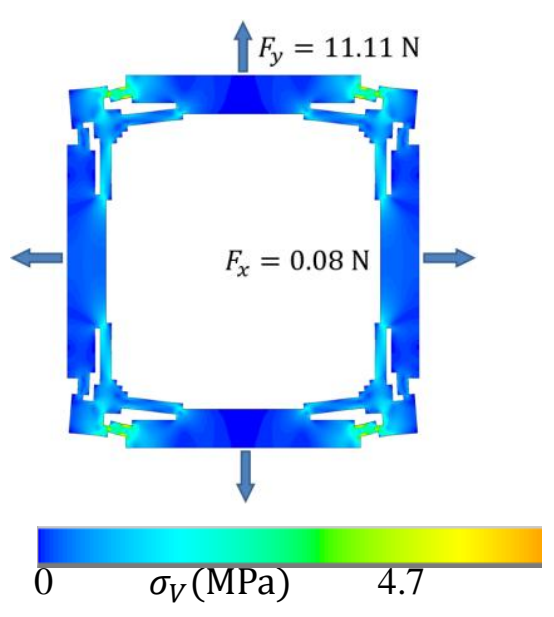

(b) TR6

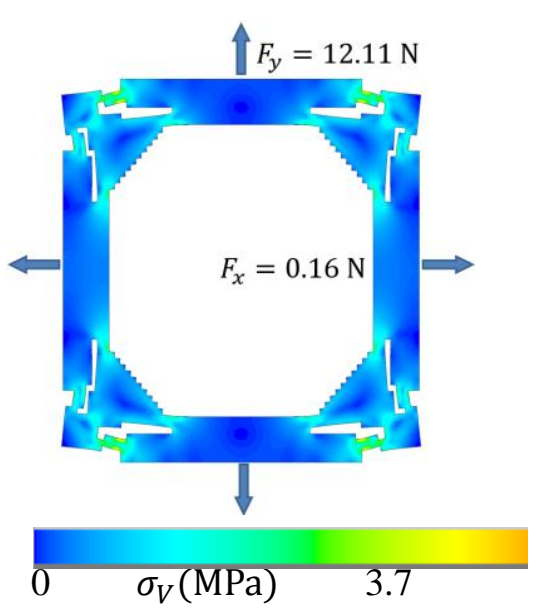

(c) TR8

Figure A.1. (Color online). FEM simulation of tensile test and resultant contour of von Mises stress $\sigma_{V}$ in the central cell and relevant edge loads, for tensile test load of $F_{T}=70 \mathrm{~N}$ (in average $10 \mathrm{~N}$ per column of unit-cells in the $7 \times 7 \mathrm{PhP}$ specimen as shown in Figure 12(a)), for topologies (a) TR3, (b) TR6 and (c) TR8, (Deformations are magnified for clarity)

The von Mises stress $\sigma_{V}$ contour shows that the maximum stress in the unit-cell is significantly reduced from 24.5 MPa for compliant topology TR3 to 4.7 MPa and 3.7 MPa for stiff topologies TR6 and TR8, respectively, under the same tensile load.

For the applied tensile load $F_{T}=70 \mathrm{~N}$, a $10 \mathrm{~N}$ load is expected per column of $7 \times 7 \mathrm{PhP}$ array by the assumption of uniform load distribution. However, as confirmed by the calculated 
longitudinal loads $F_{y}$ depicted in Figure A.1, the actual longitudinal load applied to the central unit-cell is higher than the uniform magnitude of $10 \mathrm{~N}$. Moreover, a relatively small transversal load $F_{y}$ is applied to the unit-cell slightly resisting its expected transverse contraction. The nonuniform longitudinal load distribution and transversal loads are due to finite nature of the specimen and the fact that transverse displacement of upper and lower boundaries of PhP section are constrained by the uniform plate sections having much higher stiffness.

Consequently, the assumption of a uniform load distribution between $\mathrm{PhP}$ columns leads to underestimation of the elastic modulus based on measured displacements of the central unit-cell, as confirmed by the results given in Table 2 in which $\bar{E}<E_{e}$.

\section{References}

Aberg, M \& Gudmundson, P 1997, 'The usage of standard finite element codes for computation of dispersion relations in materials with periodic microstructure', The Journal of the Acoustical Society of America, vol. 102, p. 2007.

Bilal, OR \& Hussein, MI 2011, 'Ultrawide phononic band gap for combined in-plane and out-ofplane waves', Physical Review E, vol. 84, no. 6, p. 065701.

Bilal, OR \& Hussein, MI 2012, 'Topologically evolved phononic material: Breaking the world record in band gap size', Photonic and Phononic Properties of Engineered Nanostructures, International Socienty for Optics and Photonics, pp. 826911-826917.

Celli, P \& Gonella, S 2014, 'Laser-enabled experimental wavefield reconstruction in twodimensional phononic crystals', Journal of Sound and Vibration, vol. 333, no. 1, 1/6/, pp. 114123.

Delpero, T, Schoenwald, S, Zemp, A \& Bergamini, A 2016, 'Structural engineering of threedimensional phononic crystals', Journal of Sound and Vibration, vol. 363, 2/17/, pp. 156-165.

Deymier, P 2011, Acoustic metamaterials and phononic crystals, Springer,

Dong, H-W, Su, X-X \& Wang, Y-S 2014, 'Multi-objective optimization of two-dimensional porous phononic crystals', Journal of Physics D: Applied Physics, vol. 47, no. 15, p. 155301.

Giurgiutiu, V 2003, 'Lamb wave generation with piezoelectric wafer active sensors for structural health monitoring', Smart Structures and Materials, International Society for Optics and Photonics, pp. 111-122.

Guest, JK \& Smith Genut, LC 2010, 'Reducing dimensionality in topology optimization using adaptive design variable fields', International Journal for Numerical Methods in Engineering, vol. 81, no. 8, pp. 1019-1045.

Guo, X, Wei, P \& Li, L 2016, 'Dispersion relations of elastic waves in one-dimensional piezoelectric phononic crystal with mechanically and dielectrically imperfect interfaces', Mechanics of Materials, vol. 93, 2//, pp. 168-183. 
Halkjær, S, Sigmund, O \& Jensen, JS 2006, 'Maximizing band gaps in plate structures', Structural and Multidisciplinary Optimization, vol. 32, no. 4, pp. 263-275.

Hedayatrasa, S, Abhary, K \& Uddin, M 2015, 'Numerical study and topology optimization of 1D periodic bimaterial phononic crystal plates for bandgaps of low order Lamb waves', Ultrasonics, vol. 57, no. 0, pp. 104-124.

Hedayatrasa, S, Abhary, K \& Uddin, M 2016, 'On topology optimization of acoustic metamaterial lattices for locally resonant bandgaps of flexural waves', The 2nd Australasian Acoustical Societies' Conference.

Hedayatrasa, S, Abhary, K, Uddin, M \& Guest, JK 2016a, 'Optimal design of tunable phononic bandgap plates under equibiaxial stretch', Smart Mater. Struct., vol. 25, no. , 2016, p. 055025.

Hedayatrasa, S, Abhary, K, Uddin, M \& Ng, C-T 2016b, 'Optimum design of phononic crystal perforated plate structures for widest bandgap of fundamental guided wave modes and maximized inplane stiffness', Mechanics and physics of solids, vol. 89, no. , pp. 31-58.

Hussein, MI, Hulbert, GM \& Scott, RA 2005, 'Hierarchical design of phononic materials and structures', Proceedings of 2005 ASME International Mechanical Engineering Congress and $R \& D$ Expo, pp. 1-10.

Hussein, MI, Hamza, K, Hulbert, GM \& Saitou, K 2007, 'Optimal synthesis of 2D phononic crystals for broadband frequency isolation', Waves in Random and Complex Media, vol. 17, no. 4, November 2007, pp. 491-510.

Kittel, C 1986, Introduction to solid state physics, Wiley New York,

Krushynska, AO, Kouznetsova, VG \& Geers, MGD 2014, 'Towards optimal design of locally resonant acoustic metamaterials', Journal of the Mechanics and Physics of Solids, vol. 71, no. 0, pp. 179-196.

Lin, S-CS 2012, 'Acoustic metamaterials: tunable gradient-index phononic crystals for acoustic wave manipulation', Engineering Science and Mechanics, The Pennsylvania State University.

Liu, Z, Zhang, X, Mao, Y, Zhu, Y, Yang, Z, Chan, C \& Sheng, P 2000, 'Locally resonant sonic materials', Science, vol. 289, no. 5485, pp. 1734-1736.

Nemat-Nasser, S 2015a, 'Refraction characteristics of phononic crystals', Acta Mechanica Sinica, vol. 31, no. 4, pp. 481-493.

Nemat-Nasser, S 2015b, 'Anti-plane shear waves in periodic elastic composites: band structure and anomalous wave refraction', Proc. R. Soc. A, The Royal Society, p. 20150152.

Olsson Iii, RH, El-Kady, IF, Su, MF, Tuck, MR \& Fleming, JG 2008, 'Microfabricated VHF acoustic crystals and waveguides', Sensors and Actuators A: Physical, vol. 145-146, no. 0, 7//, pp. 87-93. 
Park, JH, Ma, PS \& Kim, YY 2015, 'Design of phononic crystals for self-collimation of elastic waves using topology optimization method', Structural and Multidisciplinary Optimization, vol. 51, no. 6, pp. 1199-1209.

Pratap, A, Agarwal, S \& Meyarivan, T 2002, 'A fast and elitist multiobjective genetic algorithm: NSGA-II', IEEE TRANSACTIONS ON EVOLUTIONARY COMPUTATION, vol. 6, no. 2, pp. 182-197.

Sigmund, O \& Jensen, JS 2003, 'Systematic design of phononic band-gap materials and structures by topology optimization', Phil. Trans. R. Soc. Lond, vol. 361, 2003, pp. 1001-1019.

Steven, G 2006, 'Homogenization and inverse homogenization for 3D composites of complex architecture', Engineering computations, vol. 23, no. 4, pp. 432-450.

Sutton, MA, Orteu, JJ \& Schreier, H 2009, Image correlation for shape, motion and deformation measurements: basic concepts, theory and applications, Springer Science \& Business Media,

Timoshenko, SP \& Woinowsky-Krieger, S 1959, Theory of plates and shells, McGraw-hill,

Wang, Y-F \& Wang, Y-S 2013, 'Multiple wide complete bandgaps of two-dimensional phononic crystal slabs with cross-like holes', Journal of Sound and Vibration, vol. 332, no. 8, 4/15/, pp. 2019-2037.

Zhu, H \& Semperlotti, F 2014, 'A passively tunable acoustic metamaterial lens for damage detection applications', vol. 9061, pp. 906107-906107-906109. 


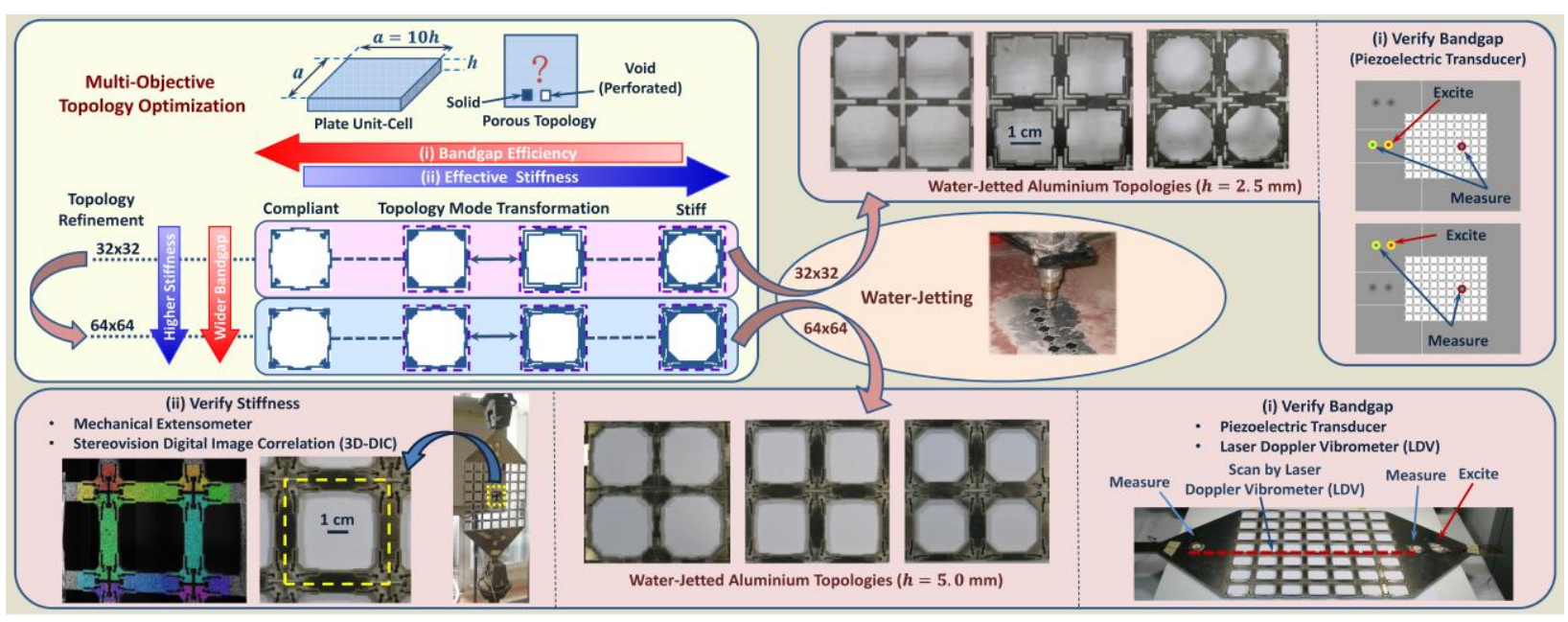

